\title{
The modular group for the total ancestor potential of Fermat simple elliptic singularities
}

\author{
Todor Milanov and Yefeng Shen
}

In a series of papers [12, 15], Krawitz, Milanov, Ruan and Shen have verified the so-called Landau-Ginzburg/Calabi-Yau (LG/ CY) correspondence for simple elliptic singularities $E_{N}^{(1,1)}(N=$ $6,7,8)$. As a by-product it was also proved that the orbifold Gromov-Witten invariants of the orbifold projective lines $\mathbb{P}_{3,3,3}^{1}$, $\mathbb{P}_{4,4,2}^{1}$ and $\mathbb{P}_{6,3,2}^{1}$ are quasi-modular forms on an appropriate modular group. While the modular group for $\mathbb{P}_{3,3,3}^{1}$ is $\Gamma(3)$, the modular groups in the other two cases were left unknown. The goal of this paper is to prove that the modular groups in the remaining two cases are, respectively, $\Gamma(4)$ and $\Gamma(6)$.

1 Introduction

1.1 Formulation of the main results

1.2 Applications to the Gromov-Witten theory

2 The total ancestor potential in singularity theory

2.1 Saito's theory

2.2 Primitive forms for simple elliptic singularities

2.3 Givental's higher-genus reconstruction formalism 
3.2 Flat coordinates

3.3 The monodromy of the flat coordinates

3.4 Monodromy of the asymptotical operator

3.5 Quasi-modularity

4 The modular groups $\Gamma(W)$

4.1 Monodromy of the hypergeometric equations

4.1.1 The resonance case

4.1.2 The non-resonance case

4.2 The group $\widetilde{\Gamma}(W)$

4.3 The Fermat $E_{6}^{(1,1)}$ case

4.4 The Fermat $E_{7}^{(1,1)}$ case

4.4.1 Monodromy of the invariant part 358

4.4.2 Monodromy of the twisted sector 358

4.5 The Fermat $E_{8}^{(1,1)}$ case

4.5.1 Monodromy of the invariant part 362

4.5.2 Monodromy of the twisted sector 363

Acknowledgments

366

References

366

\section{Introduction}

Let $W(\mathbf{x})=x_{1}^{a_{1}}+x_{2}^{a_{2}}+x_{3}^{a_{3}}$ be a Fermat polynomial whose exponents $\left(a_{1}\right.$, $\left.a_{2}, a_{3}\right)$ are given by one of the following triples $(3,3,3),(4,4,2)$ or $(6,3,2)$. Here we use the notation $\mathbf{x}=\left(x_{1}, x_{2}, x_{3}\right)$. Such a polynomial $W$ defines a hypersurface in $\mathbb{C}^{3}$ that has a simple-elliptic singularity at $\mathbf{x}=\mathbf{0} \in \mathbb{C}^{3}$ (see [24]). Moreover, the corresponding sets of vanishing cycles are known to be elliptic root systems in the sense of K. Saito (see [22]) of types, respectively, $E_{6}^{(1,1)}, E_{7}^{(1,1)}$, and $E_{8}^{(1,1)}$. We will sometimes refer to $W$ as the 
elliptic Fermat polynomial of type $E_{N}^{(1,1)}$. We assign weights $q_{i}=1 / a_{i}$ to each variable $x_{i}$, so that $W$ becomes a quasi-homogeneous polynomial of degree 1.

\subsection{Formulation of the main results}

Let $H=\mathbb{C}\left[x_{1}, x_{2}, x_{3}\right] /\left(W_{x_{1}}, W_{x_{2}}, W_{x_{3}}\right)$ be the Jacobi algebra of $W, W_{x_{i}}:=$ $\partial W / \partial x_{i}$. Given a triple $\mathbf{r}=\left(r_{1}, r_{2}, r_{3}\right)$ of non-negative integers we put $\phi_{\mathbf{r}}$ $(\mathbf{x})=x_{1}^{r_{1}} x_{2}^{r_{2}} x_{3}^{r_{3}}$. We choose a set $\mathfrak{R}$ of exponents $\mathbf{r}$, s.t., the monomials $\phi_{\mathbf{r}}(\mathbf{x})$ project to a basis of $H$. More precisely, put

$$
\mathfrak{R}=\left\{\left(r_{1}, r_{2}, r_{3}\right) \mid 0 \leq r_{i} \leq a_{i}-2\right\}
$$

It will be convenient also to decompose $\mathfrak{R}=\{\mathbf{0}, \mathbf{m}\} \sqcup \Re_{\text {tw }}$, where $\mathbf{0}:=$ $(0,0,0), \mathbf{m}:=\left(m_{1}, m_{2}, m_{3}\right), \mathfrak{R}_{\mathrm{tw}}$ corresponds to monomials of non-integral degree and $\phi_{\mathbf{m}}(\mathbf{x})$ is a monomial of degree 1 . Let us denote by $\Sigma \subset \mathbb{C}$ the set of all marginal deformations

$$
f(\sigma, \mathbf{x})=W(\mathbf{x})+\sigma \phi_{\mathbf{m}}(\mathbf{x}), \quad \sigma \in \Sigma,
$$

s.t., $f(\sigma, \mathbf{x})$ has only one critical point. The hypersurfaces

$$
X_{\sigma, \lambda}=\left\{\mathbf{x} \in \mathbb{C}^{3} \mid f(\sigma, \mathbf{x})=\lambda\right\}
$$

form a smooth fibration over $\Sigma \times(\mathbb{C} \backslash\{0\})$, while the homology (resp. cohomology) groups $H_{2}\left(X_{\sigma, \lambda} ; \mathbb{C}\right)$ (resp. $\left.H^{2}\left(X_{\sigma, \lambda} ; \mathbb{C}\right)\right)$ form a vector bundle equipped with a flat Gauss-Manin connection. We fix a reference point, say $(0,1)$, and let

$$
\mathfrak{h}=H_{2}\left(X_{0,1} ; \mathbb{C}\right), \quad \mathfrak{h}^{\vee}=H^{2}\left(X_{0,1} ; \mathbb{C}\right)
$$

be the reference fibers. The parallel transport around $\lambda=0$ induces a monodromy transformation $J \in \mathrm{GL}(\mathfrak{h})$, which commutes with the monodromy action of $\pi_{1}(\Sigma)$ on $\mathfrak{h}$. Moreover, $J$ has finite order, so it is a diagonalizable transformation whose eigenvalues are roots of unity of the form $e^{-2 \pi \sqrt{-1} d}$, $0 \leq d<1$. In particular, if we denote by $\mathfrak{h}_{0}$ the $J$-invariant subspace and by $\mathfrak{h}_{\neq 0}$ the direct sum of all eigen subspaces of $J$ with eigenvalue $\neq 1$, i.e., 
$d \neq 0$, then the monodromy representation has the form

$$
\rho: \pi_{1}(\Sigma) \rightarrow \mathrm{GL}\left(\mathfrak{h}_{0}\right) \oplus \mathrm{GL}\left(\mathfrak{h}_{\neq 0}\right)
$$

Put $\rho=\left(\rho_{0}, \rho_{\neq 0}\right)$ and let

$$
\bar{\rho}_{\neq 0}: \pi_{1}(\Sigma) \longrightarrow \mathrm{GL}\left(\mathfrak{h}_{\neq 0}\right) /\langle J\rangle
$$

be the map induced from $\rho_{\neq 0}$. Using an explicit computation we will check that

$$
\operatorname{Ker}\left(\rho_{0}\right) \subset \operatorname{Ker}\left(\bar{\rho}_{\neq 0}\right) \text {. }
$$

It will be nice if one can find a conceptual explanation and determine if (2) is satisfied for other normal forms $W$ of the simple elliptic singularities. Using (2) we get an induced homomorphism

$$
\rho_{W}: \widetilde{\Gamma}(W) \longrightarrow \operatorname{GL}\left(\mathfrak{h}_{\neq 0}\right) /\langle J\rangle
$$

where $\widetilde{\Gamma}(W)=\operatorname{Im}\left(\rho_{0}\right)$. Put $\Gamma(W):=\operatorname{Ker}\left(\rho_{W}\right)$.

Theorem 1.1. The total ancestor potential $\mathscr{A}_{\sigma}^{W}(\hbar ; \mathbf{q})$ of the simple elliptic singularity $W$ transforms as a quasi-modular form on $\Gamma(W)$.

The definition of the total ancestor potential in singularity theory as well as the precise meaning of the quasi-modularity will be recalled later on. Our second result can be stated this way.

Theorem 1.2. If $W$ is an elliptic Fermat polynomial of type $E_{6}^{(1,1)}, E_{7}^{(1,1)}$ or $E_{8}^{(1,1)}$, then $\Gamma(W)$ is, respectively, $\Gamma(3), \Gamma(4)$ or $\Gamma(6)$.

\subsection{Applications to the Gromov-Witten theory}

The Landau-Ginzburg/Calabi-Yau (LG/CY) correspondence was proposed by Ruan [19]. In our settings it can be stated this way. A triplet of non-zero complex numbers $\left(\lambda_{1}, \lambda_{2}, \lambda_{3}\right) \in\left(\mathbb{C}^{*}\right)^{3}$ is called a diagonal symmetry 
of $W$ if

$$
W\left(\lambda_{1} x_{1}, \lambda_{2} x_{2}, \lambda_{3} x_{3}\right)=W\left(x_{1}, x_{2}, x_{3}\right)
$$

The diagonal symmetries form a group $G_{W}$. It contains the element

$$
J_{W}=\operatorname{diag}\left(e^{2 \pi \sqrt{-1} q_{1}}, e^{2 \pi \sqrt{-1} q_{2}}, e^{2 \pi \sqrt{-1} q_{3}}\right), \quad q_{i}=1 / a_{i} .
$$

The equation $W=0$ defines an elliptic curve $X_{W}$ in the weighted projective plane $\mathbb{P}^{2}\left(c_{1}, c_{2}, c_{3}\right)$, where $q_{i}=c_{i} / d$ for a common denominator $d$. The action of the group $\widetilde{G}_{W}:=G_{W} /\left\langle J_{W}\right\rangle$ on $X_{W}$ is faithful and the quotient $\mathcal{X}_{W}:=X_{W} / \widetilde{G}_{W}$ is an orbifold projective line $\mathbb{P}_{a_{1}, a_{2}, a_{3}}^{1}$. The LG/CY correspondence predicts that the GW invariants of $\mathcal{X}_{W}$ can be obtained from the so-called Fan-Jarvis-Ruan-Witten invariants (see $[7,8]$ ) of the pair $\left(W, G_{W}\right)$ via analytic continuation and a certain quantizatied symplectic transformation (cf. [19]). Chiodo-Ruan addressed the idea of using global mirror symmetry to solve the LG/CY correspondence $[4,5]$. This approach has been very successful so far, see $[4,12,15]$ for more details.

The orbifold $\mathrm{GW}$ invariants of $\mathcal{X}=\mathbb{P}_{a_{1}, a_{2}, a_{3}}^{1}$ are defined as follows. Let $\overline{\mathcal{M}}_{g, n, \beta}^{\mathcal{X}}$ be the moduli space of degree- $\beta$ stable maps from a genus- $g$ orbicurve, equipped with $n$ marked points, to $\mathcal{X}$. Here $\beta \in \operatorname{Eff}(\mathcal{X})$ where Eff $(\mathcal{X}) \subset H_{2}(\mathcal{X} ; \mathbb{Z})$ is the cone of effective curve classes. By definition the Novikov ring is the completed group algebra of $\operatorname{Eff}(\mathcal{X})$. In our case, since $H_{2}(\mathcal{X} ; \mathbb{Z})=\mathbb{Z} \cdot[\mathcal{X}]$, where $[\mathcal{X}]$ is the fundamental class of $\mathcal{X}$, we may identify the Novikov ring with the space of formal power series $\mathbb{C} \llbracket q \rrbracket$ and replace $\overline{\mathcal{M}}_{g, n, d \cdot[\mathcal{X}]}^{\mathcal{X}}$ by $\overline{\mathcal{M}}_{g, n, d}^{\mathcal{X}}$. Let us denote by $\pi$ the forgetful map, and by $\mathrm{ev}_{i}$ the evaluation at the $i$ th marked point

$$
\overline{\mathcal{M}}_{g, n} \stackrel{\pi}{\longleftarrow} \overline{\mathcal{M}}_{g, n, d}^{\mathcal{X}} \stackrel{\mathrm{ev}_{\mathrm{i}}}{\longrightarrow} I \mathcal{X}
$$

where $I \mathcal{X}$ is the inertia orbifold of $\mathcal{X}$. The moduli space is equipped with a virtual fundamental cycle $\left[\overline{\mathcal{M}}_{g, n, d}^{\mathcal{X}}\right]$, s.t., the maps

$$
\Lambda_{g, n}^{\mathcal{X}}: H_{C R}^{*}(\mathcal{X} ; \mathbb{C} \llbracket q \rrbracket)^{\otimes n} \longrightarrow H^{*}\left(\overline{\mathcal{M}}_{g, n} ; \mathbb{C}\right)
$$

defined by

$$
\Lambda_{g, n}^{\mathcal{X}}=\sum_{d=0}^{\infty} q^{d} \Lambda_{g, n, d}^{\mathcal{X}}, \quad \Lambda_{g, n, d}^{\mathcal{X}}\left(\alpha_{1}, \ldots, \alpha_{n}\right):=\pi_{*}\left(\left[\overline{\mathcal{M}}_{g, n, d}^{\mathcal{X}}\right] \cap \prod_{i=1}^{n} \operatorname{ev}_{i}^{*}\left(\alpha_{i}\right)\right)
$$


form a CohFT with state space the Chen-Ruan cohomology $H_{C R}^{*}(\mathcal{X} ; \mathbb{C} \llbracket q \rrbracket)$. The ancestor $\mathrm{GW}$ invariants of $\mathcal{X}$ are by definition the following formal series:

(4)

$$
\left\langle\tau_{k_{1}}\left(\alpha_{1}\right), \ldots, \tau_{k_{n}}\left(\alpha_{n}\right)\right\rangle_{g, n}=\sum_{d=0}^{\infty} q^{d} \int_{\overline{\mathcal{M}}_{g, n}} \Lambda_{g, n, d}^{\mathcal{X}}\left(\alpha_{1}, \ldots, \alpha_{n}\right) \psi_{1}^{k_{1}} \cdots \psi_{n}^{k_{n}},
$$

where $\psi_{i}$ is the $i$ th psi class on $\overline{\mathcal{M}}_{g, n}, \alpha_{i} \in H_{C R}^{*}(\mathcal{X} ; \mathbb{C})$, and $k_{i} \in \mathbb{Z}_{\geq 0}$. For more details on orbifold Gromov-Witten theory we refer to [3]. The total ancestor potential of $\mathcal{X}$ is by definition the following generating series:

$$
\mathscr{A}_{q}^{\mathcal{X}}(\hbar ; \mathbf{q})=\exp \left(\sum_{g, n=0}^{\infty} \hbar^{g-1}\left\langle\mathbf{q}\left(\psi_{1}\right)+\psi_{1}, \ldots, \mathbf{q}\left(\psi_{n}\right)+\psi_{n}\right\rangle_{g, n}\right),
$$

where $\mathbf{q}(z)=\sum_{k=0}^{\infty} \mathbf{q}_{k} z^{k}$ and $\left\{\mathbf{q}_{k}\right\}_{k=0}^{\infty}$ is a sequence of formal vector variables with values in $H_{\mathrm{CR}}^{*}(\mathcal{X} ; \mathbb{C})$. The generating function is a formal series in $\mathbf{q}_{0}, \mathbf{q}_{1}+1, \mathbf{q}_{2}, \ldots$

Following the ideas of Krawitz-Shen [12] and Milanov-Ruan [15], one can obtain a very precise correspondence between the total ancestor potentials $\mathscr{A}_{\sigma}^{W}$ and $\mathscr{A}_{q}^{\mathcal{X}}$ (see [16]). Let us briefly explain this correspondence. Recall that the curve

$$
E_{\sigma}=\{f(\sigma, \mathbf{x})=0\} \subset \mathbb{P}^{2}\left(c_{1}, c_{2}, c_{3}\right)
$$

is called the elliptic curve at infinity. Let us think of $\Sigma$ as a punctured $\mathbb{P}^{1}$ and let us select a puncture $p$, s.t., the $j$-invariant $j\left(E_{\sigma}\right) \rightarrow \infty$ as $\sigma \rightarrow p$. For example, if $W$ is the Fermat polynomial of type $E_{7}^{(1,1)}$, then $p=-2,2$ or $\infty$. If $W$ is the Fermat polynomial of type $E_{8}^{(1,1)}$, then $p$ is a solution to $4 p^{3}+27=0$. The main statement is that there exists a function $\pi_{B}(\sigma) / \pi_{A}(\sigma)$ (see $[16]$ ) on $\mathbb{P}^{1}$ holomorphic near $\sigma=p$, s.t., under a mirror map $q=\pi_{B}(\sigma) / \pi_{A}(\sigma)$, the total ancestor potential $\mathscr{A}_{q}^{\mathcal{X}}$ coincides with $\mathscr{A}_{\sigma}^{W}$. Let us point out that the definition of $\mathscr{A}_{\sigma}^{W}$ requires a choice of a primitive form in the sense of Saito [20]. Part of the statement is that there exists a primitive form, s.t., the identification holds. Combining the mirror symmetry theorem of [16] with Theorems 1.1 and 1.2 we get

Corollary 1.3. If $\mathcal{X}$ is one of the orbifolds $\mathbb{P}_{3,3,3}^{1}, \mathbb{P}_{4,4,2}^{1}$ or $\mathbb{P}_{6,3,2}^{1}$, then the Gromov-Witten invariants (4) are quasi-modular forms, respectively, on $\Gamma(3), \Gamma(4)$ or $\Gamma(6)$. 


\section{The total ancestor potential in singularity theory}

Let $\mathcal{S}=\Sigma \times \mathbb{C}^{\mu-1}$. We fix a coordinate system on $\mathcal{S}$, such that the coordinates of $\mathbf{s}=\left(s_{\mathbf{m}}, s_{\mathbf{r}_{1}}, \ldots, s_{\mathbf{r}_{\mu-1}}\right) \in \mathcal{S}$ are indexed by the exponents $\mathfrak{R}$ (cf. (1)) in such a way that $s_{\mathbf{m}} \in \Sigma$ and $s_{\mathbf{r}} \in \mathbb{C}$ for $\mathbf{r} \neq \mathbf{m}$. The miniversal deformation of $W$ can be given by the following function:

$$
F(\mathbf{s}, \mathbf{x})=W(\mathbf{x})+\sum_{\mathbf{r} \in \mathfrak{R}} s_{\mathbf{r}} \phi_{\mathbf{r}}(\mathbf{x}),
$$

where the domain of $F(\mathbf{s}, \mathbf{x})$ is $X:=\mathcal{S} \times \mathbb{C}^{3}$. The marginal deformations $f(\sigma, \mathbf{x})$ are obtained from $F(\mathbf{s}, \mathbf{x})$ by restricting $s_{\mathbf{m}}=\sigma$ and $s_{\mathbf{r}}=0$ for $\mathbf{r} \neq \mathbf{m}$.

It is well known (see $[11,23])$ that Saito's theory of primitive forms (cf. [20]) gives rise to a Frobenius manifold structure (cf. [6]) on $\mathcal{S}$. In this section, the goal is to recall the key points in the construction of this Frobenius manifold structure and then use the higher-genus reconstruction formalism of Givental to define the total ancestor potential of $W$.

\subsection{Saito's theory}

Let $C \subset X$ be the critical variety of $F(\mathbf{s}, \mathbf{x})$, i.e., the support of the sheaf

$$
\mathcal{O}_{C}:=\mathcal{O}_{X} /\left\langle F_{x_{1}}, F_{x_{2}}, F_{x_{3}}\right\rangle
$$

Let $q: X \rightarrow \mathcal{S}$ be the projection on the first factor. The Kodair-Spencer $\operatorname{map}\left(\mathcal{T}_{\mathcal{S}}\right.$ is the sheaf of holomorphic vector fields on $\left.\mathcal{S}\right)$

$$
\mathcal{T}_{\mathcal{S}} \longrightarrow q_{*} \mathcal{O}_{C}, \quad \partial / \partial s_{\mathbf{r}} \mapsto \partial F / \partial s_{\mathbf{r}} \bmod \left(F_{x_{1}}, F_{x_{2}}, F_{x_{3}}\right)
$$

is an isomorphism, which implies that for any $\mathbf{s} \in \mathcal{S}$, the tangent space $T_{\mathbf{S}} \mathcal{S}$ is equipped with an associative commutative multiplication $\bullet_{\mathbf{s}}$ depending holomorphically on $\mathbf{s} \in \mathcal{S}$. If in addition we have a volume form $\omega=g(\mathbf{s}, \mathbf{x}) d^{3} \mathbf{x}$, where $d^{3} \mathbf{x}=d x_{1} \wedge d x_{2} \wedge d x_{3}$ is the standard volume form; then $q_{*} \mathcal{O}_{C}$ (hence $\mathcal{T}_{\mathcal{S}}$ as well) is equipped with the residue pairing:

$$
\left(\psi_{1}, \psi_{2}\right)=\frac{1}{(2 \pi i)^{3}} \int_{\Gamma_{\epsilon}} \frac{\psi_{1}(\mathbf{s}, \mathbf{y}) \psi_{2}(\mathbf{s}, \mathbf{y})}{F_{y_{1}} F_{y_{2}} F_{y_{3}}} \omega
$$

where $\mathbf{y}=\left(y_{1}, y_{2}, y_{3}\right)$ is an unimodular coordinate system for the volume form, i.e., $\omega=d^{3} \mathbf{y}$, and $\Gamma_{\epsilon}$ is a real three-dimensional cycle supported on $\left|F_{x_{i}}\right|=\epsilon$ for $1 \leq i \leq 3$. 
Given a semi-infinite cycle

$$
\mathcal{A} \in \lim _{\longleftarrow} H_{3}\left(\mathbb{C}^{3},\left(\mathbb{C}^{3}\right)_{-m} ; \mathbb{C}\right) \cong \mathbb{C}^{\mu},
$$

where

$$
\left(\mathbb{C}^{3}\right)_{m}=\left\{\mathbf{x} \in \mathbb{C}^{3} \mid \operatorname{Re}(F(\mathbf{s}, \mathbf{x}) / z) \leq m\right\}
$$

Put

$$
J_{\mathcal{A}}(\mathbf{s}, z)=(-2 \pi z)^{-3 / 2} z d_{\mathcal{S}} \int_{\mathcal{A}} e^{F(\mathbf{s}, \mathbf{x}) / z} \omega,
$$

where $d_{\mathcal{S}}$ is the de Rham differential on $\mathcal{S}$. The oscillatory integrals $J_{\mathcal{A}}$ are by definition sections of the cotangent sheaf $\mathcal{T}_{\mathcal{S}}^{*}$.

According to Saito's theory of primitive forms [20, 24], there exists a volume form $\omega$ such that the residue pairing is flat and the oscillatory integrals satisfy a system of differential equations, which in flat-homogeneous coordinates $\mathbf{t}=\left(t_{\mathbf{r}}\right)_{\mathbf{r} \in \mathfrak{R}}$ have the form

$$
z \partial_{\mathbf{r}} J_{\mathcal{A}}(\mathbf{t}, z)=\partial_{\mathbf{r}} \bullet_{\mathbf{t}} J_{\mathcal{A}}(\mathbf{t}, z),
$$

where $\partial_{\mathbf{r}}:=\partial / \partial t_{\mathbf{r}}$ and the multiplication is defined by identifying vectors and covectors via the residue pairing. Using the residue pairing, the flat structure, and the Kodaira-Spencer isomorphism we have the following isomorphisms:

$$
T^{*} \mathcal{S} \cong T \mathcal{S} \cong \mathcal{S} \times T_{0} \mathcal{S} \cong \mathcal{S} \times H .
$$

Due to homogeneity, the integrals satisfy a differential equation with respect to the parameter $z \in \mathbb{C}^{*}$

$$
\left(z \partial_{z}+E\right) J_{\mathcal{A}}(\mathbf{t}, z)=\Theta J_{\mathcal{A}}(\mathbf{t}, z)
$$

where

$$
E=\sum_{\mathbf{r} \in \mathfrak{R}} d_{\mathbf{r}} t_{\mathbf{r}} \partial_{\mathbf{r}} \quad\left(d_{\mathbf{r}}:=\operatorname{deg} t_{\mathbf{r}}=\operatorname{deg} s_{\mathbf{r}}\right),
$$

is the Euler vector field and $\Theta$ is the so-called Hodge grading operator

$$
\Theta: \mathcal{T}_{S}^{*} \rightarrow \mathcal{T}_{S}^{*}, \quad \Theta\left(d t_{\mathbf{r}}\right)=\left(\frac{1}{2}-d_{\mathbf{r}}\right) d t_{\mathbf{r}} .
$$


The compatibility of systems (10) and (11) implies that the residue pairing, the multiplication, and the Euler vector field give rise to a conformal Frobenius structure of conformal dimension 1. We refer to Dubrovin [6] for the definition and more details on Frobenius structures.

\subsection{Primitive forms for simple elliptic singularities}

The classification of primitive forms in general is a very difficult problem. In the case of simple elliptic singularities however, all primitive forms are known (see [20]). They are given by $\omega=d^{3} \mathbf{x} / \pi_{A}(\sigma)$, where $\pi_{A}(\sigma)$ is a period of the elliptic curve at infinity. Let us recall also that $\pi_{A}(\sigma)$ can be expressed in terms of a period of the Gelfand-Lerey form $d^{3} \mathbf{x} / d f$ as follows. We embed $\mathbb{C}^{3}$ in the weighted projective space $\mathbb{P}^{3}\left(1, c_{1}, c_{2}, c_{3}\right)$ via $x_{i}=X_{i} / X_{0}^{c_{i}}, i=1,2,3$. The Zariski closure of the Milnor fiber $X_{\sigma, 1}$ is $\bar{X}_{\sigma, 1}=X_{\sigma, 1} \cup E_{\sigma}$, therefore we have a tube map

$$
L: H_{1}\left(E_{\sigma} ; \mathbb{Z}\right) \rightarrow H_{2}\left(X_{\sigma, 1} ; \mathbb{Z}\right)
$$

which allows us to write

$$
\pi_{A}(\sigma):=\int_{L(A)} \frac{d^{3} \mathbf{x}}{d f}=2 \pi \sqrt{-1} \int_{A} \operatorname{Res}_{E_{\sigma}} \frac{d^{3} \mathbf{x}}{d f} .
$$

Let us point out that when $\sigma=0$ the image of the tube map $L$ is precisely $\mathfrak{h}_{0}$ and the monodromy representation $\rho_{0}$ coincides with the monodromy representation of the elliptic pencil $E_{\sigma}, \sigma \in \Sigma$.

\subsection{Givental's higher-genus reconstruction formalism}

Following Givental we introduce the vector space $\mathcal{H}=H((z))$ of formal Laurent series in $z^{-1}$ with coefficients in $H$, equipped with the symplectic structure

$$
\Omega(f(z), g(z))=\operatorname{res}_{z=0}(f(-z), g(z)) d z
$$

Using the polarization $\mathcal{H}=\mathcal{H}_{+} \oplus \mathcal{H}_{-}$, where $\mathcal{H}_{+}=H[z]$ and $\mathcal{H}_{-}=H$ $\left[\left[z^{-1}\right]\right] z^{-1}$ we identify $\mathcal{H}$ with the cotangent bundle $T^{*} \mathcal{H}_{+}$. The goal in this subsection is to define the total ancestor potential of $W$.

2.3.1. The stationary phase asymptotics. We fix a primitive form $\omega=d^{3} \mathbf{x} / \pi_{A}(\sigma)$ and let $\left\{t_{\mathbf{r}}\right\}$ be flat coordinates, defined near $\mathbf{s}=0$, s.t., 
under the Kodaira-Spencer isomorphism $T_{0} \mathcal{S} \cong H$ we have $\partial_{\mathbf{r}}=\phi_{\mathbf{r}}(\mathbf{x})$. Since, $\pi_{A}$ is a multi-valued analytic function on $\mathcal{S}$, the flat coordinates $t_{\mathbf{r}}$ are also multi-valued analytic functions on $\mathcal{S}$.

Let $\mathbf{s} \in \mathcal{S}$ be a semi-simple point, i.e., the critical values $\left\{u_{i}(\mathbf{s})\right\}_{i=1}^{\mu}$ of $F(\mathbf{s}, \mathbf{x})$ form locally near $\mathbf{s}$ a coordinate system. Let us also fix a path from $0 \in S$ to s, so that we have a fixed branch of the flat coordinates. Then we have an isomorphism

$$
\Psi_{\mathbf{s}}: \mathbb{C}^{\mu} \rightarrow H, \quad e_{i} \mapsto \sqrt{\Delta_{i}} \partial_{u_{i}}=\sqrt{\Delta_{i}} \sum_{\mathbf{r} \in \mathfrak{R}} \frac{\partial u_{i}}{\partial t_{\mathbf{r}}} \phi_{\mathbf{r}}
$$

where $\Delta_{i}$ is determined by $\left(\partial / \partial u_{i}, \partial / \partial u_{j}\right)=\delta_{i j} / \Delta_{i}$. It is well known that $\Psi_{s}$ diagonalizes the Frobenius multiplication and the residue pairing, i.e.,

$$
e_{i} \bullet e_{j}=\sqrt{\Delta_{i}} e_{i} \delta_{i, j}, \quad\left(e_{i}, e_{j}\right)=\delta_{i j}
$$

Let $\mathcal{S}_{\mathrm{sS}}$ be the set of all semi-simple points. The complement $\mathcal{K}=\mathcal{S} \backslash \mathcal{S}_{\mathrm{sS}}$ is an analytic hypersurface also known as the caustic. It corresponds to deformations, s.t., $F(\mathbf{s}, \mathbf{x})$ has at least one non-Morse critical point. By definition, we get a multi-valued analytic map

$$
\mathcal{S}_{\mathrm{sS}} \rightarrow \operatorname{Hom}_{\mathbb{C}}\left(\mathbb{C}^{\mu}, H\right), \quad \mathbf{s} \mapsto \Psi_{\mathrm{s}}
$$

The system of differential equations (10) and (11) admits a unique formal asymptotical solution of the type

$$
\Psi_{\mathbf{s}} R_{\mathbf{s}}(z) e^{U_{\mathbf{s}} / z}, \quad R_{\mathbf{s}}(z)=1+z R_{\mathbf{s}, 1}+z^{2} R_{\mathbf{s}, 2}+\cdots
$$

where $U_{\mathbf{s}}$ is a diagonal matrix with entries $u_{1}(\mathbf{s}), \ldots, u_{\mu}(\mathbf{s})$ on the diagonal and $R_{\mathbf{s}, k} \in \operatorname{Hom}_{\mathbb{C}}\left(\mathbb{C}^{\mu}, \mathbb{C}^{\mu}\right)$. Alternatively this formal solution coincides with the stationary phase asymptotics of the following integrals. Let $\mathcal{B}_{i}$ be the semi-infinite cycle of the type (7) consisting of all points $\mathbf{x} \in \mathbb{C}^{3}$ such that the gradient trajectories of $-\operatorname{Re}(F(\mathbf{s}, \mathbf{x}) / z)$ flow into the critical value $u_{i}(\mathbf{s})$. Then

$$
(-2 \pi z)^{-3 / 2} z d_{S} \int_{\mathcal{B}_{i}} e^{F(\mathbf{s}, \mathbf{x}) / z} \omega \sim e^{u_{i}(\mathbf{s}) / z} \Psi_{\mathbf{s}} R_{\mathbf{S}}(z) e_{i} \quad \text { as } \quad z \rightarrow 0
$$

We refer to $[9,10]$ for more details and proofs. 
2.3.2. The quantization formalism. Let us fix a Darboux coordinate system on $\mathcal{H}$ given by the linear functions $q_{k}^{\mathbf{r}}, p_{k, \mathbf{r}}$ defined as follows:

$$
\mathbf{f}(z)=\sum_{k=0}^{\infty} \sum_{\mathbf{r} \in \mathfrak{R}}\left(q_{k}^{\mathbf{r}} \phi_{\mathbf{r}} z^{k}+p_{k, \mathbf{r}} \phi^{\mathbf{r}}(-z)^{-k-1}\right) \in \mathcal{H}
$$

where $\left\{\phi^{\mathbf{r}}\right\}_{\mathbf{r} \in \mathfrak{R}}$ is a basis of $H$ dual to $\left\{\phi_{\mathbf{r}}\right\}_{\mathbf{r} \in \mathfrak{R}}$ with respect to the residue pairing.

If $R=e^{A(z)}$, where $A(z)$ is an infinitesimal symplectic transformation, then we define $(R)^{\wedge}$ as follows. Since $A(z)$ is infinitesimal symplectic, the map $\mathbf{f} \in \mathcal{H} \mapsto A \mathbf{f} \in \mathcal{H}$ defines a Hamiltonian vector field with Hamiltonian given by the quadratic function $h_{A}(\mathbf{f})=\frac{1}{2} \Omega(A \mathbf{f}, \mathbf{f})$. By definition, the quantization of $e^{A}$ is given by the differential operator $e^{\left(h_{A}\right)^{\wedge}}$, where the quadratic Hamiltonians are quantized according to the following rules:

$$
\begin{aligned}
\left(p_{k^{\prime}, \mathbf{r}^{\prime}} p_{k^{\prime \prime}, \mathbf{r}^{\prime \prime}}\right)^{\wedge} & =\hbar \frac{\partial^{2}}{\partial q_{k^{\prime}}^{\mathbf{r}^{\prime}} \partial q_{k^{\prime \prime}}^{\mathbf{r}^{\prime \prime}}}, \quad\left(p_{k^{\prime}, \mathbf{r}^{\prime}} q_{k^{\prime \prime}}^{\mathbf{r}^{\prime \prime}}\right)^{\wedge}=\left(q_{k^{\prime \prime}}^{\mathbf{r}^{\prime \prime}} p_{k^{\prime}, \mathbf{r}^{\prime}}\right)^{\wedge}=q_{k^{\prime \prime}}^{\mathbf{r}^{\prime \prime}} \frac{\partial}{\partial q_{k^{\prime}}^{\mathbf{r}^{\prime}}} \\
\left(q_{k^{\prime}}^{\mathbf{r}^{\prime}} q_{k^{\prime \prime}}^{\mathbf{r}^{\prime \prime}}\right. & \wedge \\
& =q_{k^{\prime}}^{\mathbf{r}^{\prime}} q_{k^{\prime \prime}}^{\mathbf{r}^{\prime \prime}} / \hbar .
\end{aligned}
$$

Note that the quantization defines a projective representation of the Poisson-Lie algebra of quadratic Hamiltonians

$$
\left[\left(h_{1}\right)^{\wedge},\left(h_{2}\right)^{\wedge}\right]=\left(\left\{h_{1}, h_{2}\right\}\right)^{\wedge}+C\left(h_{1}, h_{2}\right)
$$

where $h_{1}$ and $h_{2}$ are quadratic Hamiltonians and the values of the cocycle $C$ on a pair of Darboux monomials is non-zero only in the following cases:

$$
C\left(p_{k^{\prime}, \mathbf{r}^{\prime}} p_{k^{\prime \prime}, \mathbf{r}^{\prime \prime}}, q_{k^{\prime}}^{\mathbf{r}^{\prime}} q_{k^{\prime \prime}}^{\mathbf{r}^{\prime \prime}}\right)= \begin{cases}1 & \text { if }\left(k^{\prime}, \mathbf{r}^{\prime}\right) \neq\left(k^{\prime \prime}, \mathbf{r}^{\prime \prime}\right), \\ 2 & \text { if }\left(k^{\prime}, \mathbf{r}^{\prime}\right)=\left(k^{\prime \prime}, \mathbf{r}^{\prime \prime}\right) .\end{cases}
$$

The quantized quadratic Hamiltonians act naturally on the Fock space of formal power series

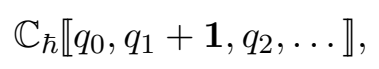

where $\mathbb{C}_{\hbar}:=\mathbb{C}((\hbar))$ is the field of formal Laurent series in $\hbar$. 
2.3.3. The total ancestor potential. By definition, the KontsevichWitten tau-function is the following generating series:

$$
\mathcal{D}_{\mathrm{pt}}(\hbar ; q(z))=\exp \left(\sum_{g, n} \frac{1}{n !} \hbar^{g-1} \int_{\overline{\mathcal{M}}_{g, n}} \prod_{i=1}^{n}\left(q\left(\psi_{i}\right)+\psi_{i}\right)\right)
$$

where $q(z)=\sum_{k} q_{k} z^{k},\left(q_{0}, q_{1}, \ldots\right)$ are formal variables, $\psi_{i}(1 \leq i \leq n)$ are the first Chern classes of the cotangent line bundles on $\overline{\mathcal{M}}_{g, n}$. The function is interpreted as a formal series in $q_{0}, q_{1}+\mathbf{1}, q_{2}, \ldots$, whose coefficients are Laurent series in $\hbar$.

Let $\mathbf{s} \in \mathcal{S}_{\mathrm{ss}}$ be a semi-simple point. Although we already fixed a Darboux coordinate system on $\mathcal{H}$, we need yet another one. Namely, under the identification $\Psi_{\mathbf{s}}: \mathbb{C}^{\mu} \rightarrow H$ the standard orthonormal basis $\left\{e_{i}\right\}_{i=1}^{\mu}$ of $\mathbb{C}^{\mu}$ gives rise to Darboux coordinates ${ }^{\mathrm{i}} q_{k},{ }_{\mathrm{i}} p_{k}$ on $\mathcal{H}$ defined by

$$
\mathbf{f}(z)=\sum_{k=0}^{\infty} \sum_{i=1}^{\mu}\left({ }^{\mathrm{i}} q_{k} e_{i} z^{k}+{ }_{\mathrm{i}} p_{k} e_{i}(-z)^{-k-1}\right) .
$$

Let us denote by $\widehat{\Psi}_{\mathrm{s}}$ the isomorphism of Fock spaces (the tensor product being completed appropriately)

$$
\widehat{\Psi}_{\mathbf{s}}: \otimes_{i=1}^{\mu} \mathbb{C}_{\hbar} \llbracket \llbracket^{\mathrm{i}} q_{0},{ }^{\mathrm{i}} q_{1} \sqrt{\Delta_{i}(\mathbf{s})}+1,{ }^{\mathrm{i}} q_{2}, \ldots \rrbracket \rightarrow \mathbb{C}_{\hbar} \llbracket q_{0}, q_{1}+\mathbf{1}, q_{2}, \ldots \rrbracket,
$$

induced by the change of the Darboux coordinates. More precisely, recalling that

$$
\Psi_{\mathbf{s}} e_{i}=\sqrt{\Delta_{i}(\mathbf{s})} \sum_{\mathbf{r} \in \mathfrak{R}} \frac{\partial u_{i}}{\partial t_{\mathbf{r}}} \phi_{\mathbf{r}}
$$

we get that the map $\widehat{\Psi}_{\mathrm{s}}$ amounts to the substitutions

$$
{ }^{i} q_{k} \sqrt{\Delta_{i}(\mathbf{s})} \mapsto \sum_{\mathbf{r} \in \mathfrak{R}} \frac{\partial u_{i}}{\partial t_{\mathbf{r}}} q_{k}^{\mathbf{r}}
$$

The key property of $\widehat{\Psi}_{\mathbf{s}}$, which is equivalent to the definition (16), is that $\widehat{\Psi}_{\mathbf{s}}$ intertwines the quantizations corresponding to the two Darboux coordinate systems

$$
(h)^{\wedge} \widehat{\Psi}_{\mathbf{s}}=\widehat{\Psi}_{\mathbf{s}}(h)^{\wedge}
$$

for all quadratic functions $h$ on $\mathcal{H}$. 
Motivated by Gromov-Witten theory Givental introduced the notion of the total ancestor potential of a semi-simple Frobenius structure (see [9, 10]). In our settings the definition takes the form

$$
\mathscr{A}_{\mathbf{s}}(\hbar ; \mathbf{q}):=\widehat{\Psi_{\mathbf{s}}}\left(R_{\mathbf{s}}\right)^{\wedge}\left(e^{U_{\mathbf{s}} / z}\right)^{\wedge} \prod_{i=1}^{\mu} \mathcal{D}_{\mathrm{pt}}\left(\hbar \Delta_{i}(\mathbf{s}) ;{ }^{i} \mathbf{q}(z) \sqrt{\Delta_{i}(\mathbf{s})}\right)
$$

where

$$
\mathbf{q}(z)=\sum_{k=0}^{\infty} \sum_{\mathbf{r} \in \mathfrak{R}} q_{k}^{\mathbf{r}} z^{k} \phi_{\mathbf{r}}, \quad{ }^{i} \mathbf{q}(z)=\sum_{k=0}^{\infty}{ }^{i} q_{k} z^{k}
$$

\section{Modularity and monodromy}

The flat coordinates are multi-valued analytic functions on $\mathcal{S}$. In this section, we will compute their monodromy under analytic continuation. Once this task is completed the proof of Theorem 1.1 will be easy.

\subsection{Picard-Fuchs equations}

We consider the so-called geometric sections (see [2])

$$
\Phi_{\mathbf{r}}(\sigma, \lambda):=\int \mathbf{x}^{\mathbf{r}} \frac{d^{3} \mathbf{x}}{d f} \in H^{2}\left(X_{\sigma, \lambda} ; \mathbb{C}\right),
$$

where $\mathbf{r}=\left(r_{1}, r_{2}, r_{3}\right), r_{i} \in \mathbb{Z}_{\geq 0}$, and $\mathbf{x}^{\mathbf{r}}:=\phi_{\mathbf{r}}=x_{1}^{r_{1}} x_{2}^{r_{2}} x_{3}^{r_{3}}$. The geometric sections (18) with all $\mathbf{r} \in \mathfrak{R}$ give rise to a trivialization of the vanishing cohomology bundle. The Gauss-Manin connection corresponds to a system of Fuchsian differential equations known as Picard-Fuchs equations. It is enough to solve this system at $\lambda=1$, because the homogeneity of $f(\sigma, x)$ yields the following simple relation:

$$
\Phi_{\mathbf{r}}(\sigma, \lambda)=\lambda^{\operatorname{deg}(\mathbf{r})} \Phi_{\mathbf{r}}(\sigma, 1)
$$

where $\operatorname{deg}(\mathbf{r}):=\operatorname{deg}\left(\mathbf{x}^{\mathbf{r}}\right)=\sum_{i} r_{i} q_{i}$. The Picard-Fuchs equations have the form

$$
\partial_{\sigma} \Phi_{\mathbf{r}}(\sigma, 1)=\sum_{\mathbf{r}^{\prime} \in \Re} G_{\mathbf{r}, \mathbf{r}^{\prime}}(\sigma) \Phi_{\mathbf{r}^{\prime}}(\sigma, 1)
$$


where $\mathfrak{G}=\left(G_{\mathbf{r}, \mathbf{r}^{\prime}}(\sigma)\right)$ is a square matrix of size $\mu=|\mathfrak{R}|$, whose entries are holomorphic functions on $\Sigma$. Let us denote by $\mathfrak{F}=\left(F_{\mathbf{r}^{\prime}, \mathbf{r}^{\prime \prime}}(\sigma)\right)$ a fundamental solution to the above system, i.e., $\mathfrak{F}$ is a non-degenerate matrix satisfying $\partial_{\sigma} \mathfrak{F}=\mathfrak{G} \cdot \mathfrak{F}$. We have

$$
\Phi_{\mathbf{r}}(\sigma, \lambda)=\lambda^{\operatorname{deg}(\mathbf{r})} \sum_{\mathbf{r}^{\prime} \in \Re} F_{\mathbf{r}, \mathbf{r}^{\prime}}(\sigma) A_{\mathbf{r}^{\prime}},
$$

where $A_{\mathbf{r}^{\prime}}, \mathbf{r}^{\prime} \in \mathfrak{R}$ are multi-valued flat sections. The system (19) is blockdiagonal in the following sense:

$$
G_{\mathbf{r}^{\prime}, \mathbf{r}^{\prime \prime}} \neq 0 \quad \Rightarrow \quad \operatorname{deg}\left(\mathbf{r}^{\prime}\right)-\operatorname{deg}\left(\mathbf{r}^{\prime \prime}\right) \in \mathbb{Z} .
$$

Therefore the matrix $\mathfrak{F}$ is also block-diagonal. Since analytic continuation around $\lambda=0$ corresponds to the classical monodromy transformation $J$, we get that the vectors $\left\{A_{\mathbf{r}}\right\}_{\mathbf{r} \in \Re}$ give an eigenbasis of $\mathfrak{h}^{\vee}$, i.e., $J\left(A_{\mathbf{r}}\right)=$ $e^{-2 \pi \sqrt{-1} \operatorname{deg}(\mathbf{r})} A_{\mathbf{r}}$.

\subsection{Flat coordinates}

We follow the idea of [15], except that we will avoid the use of explicit formulas. It is convenient to introduce the following multi-index notation. We will be interested in sequences $\kappa=\left(\kappa_{\mathbf{r}}\right)_{\mathbf{r} \in \mathfrak{R} \backslash\{\mathbf{m}\}}$, where $\kappa_{\mathbf{r}}$ are nonnegative integers. Recall that $d_{\mathbf{r}}=\operatorname{deg}\left(s_{\mathbf{r}}\right)=1-\operatorname{deg}\left(\mathbf{x}^{\mathbf{r}}\right)$, we put

$$
\mathbf{x}^{\kappa}:=\prod_{\mathbf{r} \in \mathfrak{R} \backslash\{\mathbf{m}\}}\left(\mathbf{x}^{\mathbf{r}}\right)^{\kappa_{\mathbf{r}}}, \quad \frac{\mathbf{s}^{\kappa}}{\kappa !}:=\prod_{\mathbf{r} \in \mathfrak{R} \backslash\{\mathbf{m}\}} \frac{s_{\mathbf{r}}^{\kappa_{\mathbf{r}}}}{\kappa_{\mathbf{r}} !}, \quad d_{\kappa}:=\operatorname{deg}\left(\mathbf{s}^{\kappa}\right)=\sum_{\mathbf{r} \in \mathfrak{R} \backslash\{\mathbf{m}\}} \kappa_{\mathbf{r}} d_{\mathbf{r}},
$$

Let us define a block-diagonal matrix $C=\left(C_{\mathbf{r}, \mathbf{r}^{\prime}}(s)\right)_{\mathbf{r}, \mathbf{r}^{\prime} \in \mathfrak{R}}$, whose entries are holomorphic functions on $\mathcal{S}$

$$
C_{\mathbf{r}, \mathbf{r}^{\prime}}(\mathbf{s})=\sum_{\kappa: d_{\kappa}=d_{\mathbf{r}^{\prime}}} c_{\mathbf{r}, \kappa}(\sigma) \frac{\mathbf{s}^{\kappa}}{\kappa !},
$$

where the functions $c_{\mathbf{r}, \kappa}(\mathbf{s})$ are defined from the identity

$$
(-2 \pi)^{-\frac{3}{2}}\left(\int_{0}^{-\infty} e^{\lambda} \lambda^{\operatorname{deg}\left(\mathbf{x}^{\kappa}\right)+1} d \lambda\right) \int \mathbf{x}^{\kappa} \frac{d^{3} \mathbf{x}}{d f}=\sum_{\mathbf{r} \in \mathfrak{R}} c_{\mathbf{r}, \kappa}(\sigma) \Phi_{\mathbf{r}}(\sigma, 1) .
$$

The integration path in the first integral is the negative real axis and the second one is interpreted as a cohomology class in $H^{2}\left(X_{\sigma, 1} ; \mathbb{C}\right)$. The identity 
is obtained by performing successively integration by parts until the degree of the monomial $\mathbf{x}^{\kappa}$ is reduced to some number in the interval $[0,1]$. In particular, since each integration by parts decreases the degree by an integer number, the sum on the right-hand side (RHS) is over all $\mathbf{r} \in \mathfrak{R}$, s.t., $d_{\mathbf{r}}-$ $d_{\kappa} \in \mathbb{Z}$. It follows that the matrix $C$ is block-diagonal. Given cycles $\alpha_{\mathbf{r}} \in \mathfrak{h}$, $\mathbf{r} \in \mathfrak{R}$, we define the following multi-valued analytic functions on $\mathcal{S}$ :

$$
\begin{aligned}
t_{\mathbf{m}}(\mathbf{s}) & =\frac{1}{\pi_{A}}\left(C_{0, \mathbf{m}}(\mathbf{s})\left\langle\Phi_{0}(\sigma, 1), \alpha_{\mathbf{m}}\right\rangle+C_{\mathbf{m}, \mathbf{m}}(\mathbf{s})\left\langle\Phi_{m}(\sigma, 1), \alpha_{\mathbf{m}}\right\rangle\right), \\
t_{0}(\mathbf{s}) & =\frac{1}{\pi_{A}}\left(C_{\mathbf{m}, 0}(\mathbf{s})\left\langle\Phi_{\mathbf{m}}(\sigma, 1), \alpha_{0}\right\rangle+C_{0,0}(\mathbf{s})\left\langle\Phi_{0}(\sigma, 1), \alpha_{0}\right\rangle\right), \\
t_{\mathbf{r}}(\mathbf{s}) & =\frac{1}{\pi_{A}}\left(\sum_{\mathbf{r} \in \mathfrak{R}: d_{\mathbf{r}^{\prime}}=d_{\mathbf{r}}} C_{\mathbf{r}^{\prime}, \mathbf{r}}(\mathbf{s})\left\langle\Phi_{\mathbf{r}^{\prime}}(\sigma, 1), \alpha_{\mathbf{r}}\right\rangle\right), \quad \mathbf{r} \in \mathfrak{R}_{\mathrm{tw}} .
\end{aligned}
$$

Note that by definition $C_{\mathbf{m}, \mathbf{m}}(\mathbf{s})=0$ and $C_{0, \mathbf{m}}(\mathbf{s})$ is a constant independent of $\mathbf{s}$.

Proposition 3.1. There are cycles $\left\{\alpha_{\mathbf{r}}\right\}_{\mathbf{r} \in \mathfrak{R}}$ that form an eigenbasis for the classical monodromy J, s.t.,

(i) The functions $t_{\mathbf{r}}(\mathbf{s}), \mathbf{r} \in \mathfrak{R}$ form a flat coordinate system on $\mathcal{S}$.

(ii) We have $\partial_{0}=1$ and $\left(\partial_{0}, \partial_{\mathbf{m}}\right)=1$, where $\partial_{\mathbf{r}}:=\partial / \partial t_{\mathbf{r}}$.

(iii) The following identity holds (compare with the definition of $t_{0}(\mathbf{s})$ ):

$$
\begin{aligned}
& \frac{1}{2} \sum_{\mathbf{r}^{\prime}, \mathbf{r}^{\prime \prime} \in \mathfrak{R}}\left(\partial_{\mathbf{r}^{\prime}}, \partial_{\mathbf{r}^{\prime \prime}}\right) t_{\mathbf{r}^{\prime}} t_{\mathbf{r}^{\prime \prime}} \\
& \quad=\frac{1}{\pi_{A}}\left(C_{\mathbf{m}, 0}(\mathbf{s})\left\langle\Phi_{\mathbf{m}}(\sigma, 1), \alpha_{\mathbf{m}}\right\rangle+C_{0,0}(\mathbf{s})\left\langle\Phi_{0}(\sigma, 1), \alpha_{\mathbf{m}}\right\rangle\right)
\end{aligned}
$$

Proof. Let $\sigma \in \Sigma$ be an arbitrary point. We fix a path in $\Sigma$ from 0 to $\sigma$. Our goal is to construct flat coordinates in a neighborhood of $\sigma$. Given a basis of cycles $\left\{\alpha_{\mathbf{r}}\right\}_{\mathbf{r} \in \mathfrak{R}}$ we denote by $\alpha_{\mathbf{r}}^{\sigma, 1} \in H_{2}\left(X_{\sigma, 1} ; \mathbb{C}\right)$ the parallel transport of $\alpha_{\mathbf{r}}$. The polynomial $f(\sigma, \mathbf{x})$ is weighted homogeneous, so there is a natural $\mathbb{C}^{*}$-action on $\mathbb{C}^{3}$, s.t., $f(\sigma, c \cdot \mathbf{x})=c f(\sigma, \mathbf{x})$ for every $c \in \mathbb{C}^{*}$. Using this action we define

$$
\mathcal{A}_{\mathbf{r}}=\left\{(\lambda z) \cdot \mathbf{y} \mid \lambda \in(-\infty, 0], \quad \mathbf{y} \in \alpha_{\mathbf{r}}^{\sigma, 1}\right\}
$$


Note that $\mathcal{A}_{\mathbf{r}}$ is a semi-infinite cycle of the type (7), so the corresponding oscillatory integral is convergent. Using the Fubini's theorem we get

$$
\begin{aligned}
& (-2 \pi z)^{-3 / 2} \int_{\mathcal{A}_{\mathbf{r}}} e^{F(\mathbf{s}, \mathbf{x}) / z} \omega \\
& \quad=(-2 \pi z)^{-3 / 2} \int_{0}^{-\infty} e^{\lambda}(\lambda z) \int_{\alpha_{\mathbf{r}}^{\sigma, 1}} e^{\sum_{\mathbf{r} \in \mathfrak{R} \backslash\{\mathbf{m}\}} s_{\mathbf{r}} \mathbf{x}^{\mathbf{r}} \lambda^{\left(1-d_{\mathbf{r}}\right)} z^{-d_{\mathbf{r}}}} \frac{\omega}{d f} d \lambda .
\end{aligned}
$$

The exponential in the second integral on the RHS is

$$
\sum_{\kappa} \frac{\mathbf{s}^{\kappa}}{\kappa !} \mathbf{x}^{\kappa} \lambda^{\operatorname{deg}\left(\mathbf{x}^{\kappa}\right)} z^{-d_{\kappa}}
$$

where the sum is over all sequences $\kappa=\left(\kappa_{\mathbf{r}}\right)_{\mathbf{r} \in \mathfrak{R} \backslash\{\mathbf{m}\}}$ of non-negative integers. Substituting the above expansion we get

$$
(-2 \pi)^{-3 / 2} z^{-1 / 2} \sum_{\kappa}\left(\int_{0}^{-\infty} e^{\lambda} \lambda^{1+\operatorname{deg}\left(\mathbf{x}^{\kappa}\right)} d \lambda\right) \frac{\mathbf{s}^{\kappa}}{\kappa !} z^{-d_{\kappa}} \int_{\alpha_{\mathbf{r}}^{\sigma, 1}} \mathbf{x}^{\kappa} \frac{\omega}{d f} .
$$

Comparing with formula (22), we get the following formula for the oscillatory integral:

$$
J_{\mathcal{A}_{\mathbf{r}}}(\mathbf{s}, z)=z^{\frac{1}{2}} d\left(\sum_{\kappa} z^{-d_{\kappa}} \frac{\mathbf{s}^{\kappa}}{\kappa !} \sum_{\mathbf{r} \in \mathfrak{R}} \frac{c_{\mathbf{r}, \kappa}(\sigma)}{\pi_{A}}\left\langle\Phi_{\mathbf{r}}(\sigma, 1), \alpha_{\mathbf{r}}\right\rangle\right) .
$$

The oscillatory integrals $J_{\mathcal{A}_{\mathbf{r}}}(\mathbf{s}, z)$ are solutions to the differential equations (10) and (11). On the other hand, near $z=\infty$ these equations have a fundamental solution of the type $S_{\mathbf{t}}(z) z^{\Theta}$, where

$$
S_{\mathbf{t}}(z)=1+S_{\mathbf{t}, 1} z^{-1}+S_{\mathbf{t}, 2} z^{-2} \ldots, \quad S_{\mathbf{t}, k} \in \operatorname{Hom}_{\mathbb{C}}(H, H)
$$

and $\Theta$ is the Hodge grading operator (12) (see $[6,10]$ ). Therefore, we can choose the cycles $\left\{\alpha_{\mathbf{r}}\right\}$ in such a way that

$$
J_{\mathcal{A}_{\mathbf{r}}}(\mathbf{s}, z)=S_{\mathbf{t}}(z) z^{\Theta} d t_{\mathbf{r}}=z^{\frac{1}{2}-d_{\mathbf{r}}}\left(d t_{\mathbf{r}}+z^{-1} S_{\mathbf{t}, 1}\left(d t_{\mathbf{r}}\right)+\cdots\right),
$$

where $\mathbf{t}=\left(t_{\mathbf{r}}\right)$ is a flat coordinate system. Note that $d_{\mathbf{r}}>0$ for $\mathbf{r} \neq \mathbf{m}$ and $d_{\mathbf{m}}=0$. Therefore, we have

$$
J_{\mathcal{A}_{\mathbf{r}}}(\mathbf{s}, z)=z^{\frac{1}{2}-d_{\mathbf{r}}} d t_{\mathbf{r}}+z^{\frac{1}{2}-1} \delta_{\mathbf{r}, \mathbf{m}} S_{\mathbf{t}, 1}\left(d t_{\mathbf{m}}\right)+\cdots,
$$

where the dots stand for terms involving higher-order powers of $z^{-1}$. Let us choose the flat coordinates in such a way that $\partial_{0}=\mathbf{1}$ and $\left(\partial_{\mathbf{m}}, \partial_{0}\right)=1$, then 
we have

$$
S_{\mathbf{t}, 1}\left(d t_{\mathbf{m}}\right)=S_{\mathbf{t}, 1}(\mathbf{1})=\frac{1}{2} d\left(\sum_{\mathbf{r}^{\prime}, \mathbf{r}^{\prime \prime} \in \mathfrak{R}}\left(\partial_{\mathbf{r}^{\prime}}, \partial_{\mathbf{r}^{\prime \prime}}\right) t_{\mathbf{r}^{\prime}} t_{\mathbf{r}^{\prime \prime}}\right)
$$

Finally, we get

$$
J_{\mathcal{A}_{\mathbf{r}}}(\mathbf{s}, z)=z^{\frac{1}{2}} d\left(z^{-d_{\mathbf{r}}} t_{\mathbf{r}}+z^{-1} \delta_{\mathbf{r}, \mathbf{m}} \frac{1}{2} \sum_{\mathbf{r}^{\prime}, \mathbf{r}^{\prime \prime} \in \mathfrak{R}}\left(\partial_{\mathbf{r}^{\prime}}, \partial_{\mathbf{r}^{\prime \prime}}\right) t_{\mathbf{r}^{\prime}} t_{\mathbf{r}^{\prime \prime}}\right)+\cdots
$$

All statements in the proposition follow by comparing the above formula with (23).

\subsection{The monodromy of the flat coordinates}

Let us choose the fundamental matrix $\mathfrak{F}$ of the Picard-Fuchs equations (see (20)) in such a way that $\left\{\alpha_{\mathbf{r}}\right\}_{\mathbf{r} \in \mathfrak{R}}$ and $\left\{A_{\mathbf{r}}\right\}_{\mathbf{r} \in \mathfrak{R}}$ are dual bases. Furthermore, since $C_{0, \mathbf{m}} \alpha_{\mathbf{m}}$ is a tube cycle we can find $B \in H_{1}\left(E_{0} ; \mathbb{C}\right)$ such that $L(B):=$ $C_{0, \mathbf{m}} \alpha_{\mathbf{m}}$, so we have

$$
t_{\mathbf{m}}=\frac{\pi_{B}(\sigma)}{\pi_{A}(\sigma)}
$$

The flat coordinate $t_{0}$ is such that $\partial / \partial t_{0}=1$. Therefore, the coefficient in front of $s_{0}$ in $t_{0}(\mathbf{s})$ must be 1 . On the other hand, using formulas $(21)$ and (22) we get

$$
C_{0, \mathbf{m}}(\mathbf{s})=c_{0,0}, \quad C_{0,0}(\mathbf{s})=c_{0,0} s_{0}+\cdots
$$

where the dots stand for at least quadratic terms in s. It follows that $L(A)=$ $C_{0, \mathbf{m}} \alpha_{0}$.

Let $\gamma$ be a loop in $\Sigma$ based at the reference point $\sigma=0$. Let us denote by $\left[\rho_{\neq 0}(\gamma)\right]_{\mathbf{r}, \mathbf{r}^{\prime}}$ the matrix of the linear operator $\rho_{\neq 0}(\gamma)$ in the basis $\left\{\alpha_{r}\right\}_{\mathbf{r} \in \Re_{\mathrm{tw}}}$, i.e.,

$$
\rho_{\neq 0}(\gamma)\left(\alpha_{\mathbf{r}}\right)=\sum_{\mathbf{r}^{\prime} \in \Re_{\mathrm{tw}}}\left[\rho_{\neq 0}(\gamma)\right]_{\mathbf{r}^{\prime}, \mathbf{r}} \alpha_{\mathbf{r}^{\prime}}
$$

Since the monodromy representation $\rho_{\neq 0}$ commutes with the classical monodromy $J$ and $\left\{\alpha_{\mathbf{r}}\right\}$ is an eigenbasis for $J$, the matrix $\left[\rho_{\neq 0}(\gamma)\right]$ is block 
diagonal

$$
\left[\rho_{\neq 0}(\gamma)\right]_{\mathbf{r}^{\prime}, \mathbf{r}} \neq 0 \Rightarrow d_{\mathbf{r}^{\prime}}=d_{\mathbf{r}}
$$

Similarly let us denote by $\left[\rho_{0}(\gamma)\right]$ the matrix of $\rho_{0}(\gamma)$ in the basis $\left\{\alpha_{\mathbf{m}}, \alpha_{0}\right\}$

$$
\begin{aligned}
\rho_{0}(\gamma)\left(\alpha_{\mathbf{m}}\right) & =\left[\rho_{0}(\gamma)\right]_{\mathbf{m}, \mathbf{m}} \alpha_{\mathbf{m}}+\left[\rho_{0}(\gamma)\right]_{0, \mathbf{m}} \alpha_{0} \\
\rho_{0}(\gamma)\left(\alpha_{0}\right) & =\left[\rho_{0}(\gamma)\right]_{\mathbf{m}, 0} \alpha_{\mathbf{m}}+\left[\rho_{0}(\gamma)\right]_{0,0} \alpha_{0} .
\end{aligned}
$$

The space $\mathfrak{h}_{0} \cong H_{1}\left(E_{0}, \mathbb{C}\right)$ is equipped with a symplectic form that comes from the intersection pairing. By continuity, the linear transformation $\rho_{0}(\gamma)$ is a symplectic transformation, i.e., the matrix

$$
\left[\begin{array}{ll}
a & b \\
c & d
\end{array}\right]:=\left[\rho_{0}(\gamma)\right]^{T}=\left[\begin{array}{ll}
{\left[\rho_{0}(\gamma)\right]_{\mathbf{m}, \mathbf{m}}} & {\left[\rho_{0}(\gamma)\right]_{0, \mathbf{m}}} \\
{\left[\rho_{0}(\gamma)\right]_{\mathbf{m}, 0}} & {\left[\rho_{0}(\gamma)\right]_{0,0}}
\end{array}\right] \in \mathrm{SL}_{2}(\mathbb{C})
$$

An immediate corollary of Proposition 3.1 is the transformation rule for the flat coordinates under the analytic continuation along $\gamma$.

Corollary 3.2. The analytic continuation along a loop $\gamma$ transforms the flat coordinates as follows:

$$
\begin{aligned}
\tilde{t}_{\mathbf{m}} & =\frac{a t_{\mathbf{m}}+b}{c t_{\mathbf{m}}+d}, \\
\widetilde{t}_{0} & =t_{0}+\frac{c}{2\left(c t_{\mathbf{m}}+d\right)} \sum_{\mathbf{r}^{\prime}, \mathbf{r}^{\prime \prime} \in \Re_{\mathrm{tw}}}\left(\partial_{\mathbf{r}^{\prime}}, \partial_{\mathbf{r}^{\prime \prime}}\right) t_{\mathbf{r}^{\prime}} t_{\mathbf{r}^{\prime \prime}} \\
\widetilde{t}_{\mathbf{r}} & =\frac{1}{c t_{\mathbf{m}}+d} \sum_{\mathbf{r}^{\prime} \in \Re_{\mathrm{tw}}: d_{\mathbf{r}^{\prime}}=d_{\mathbf{r}}}\left[\rho_{\neq 0}(\gamma)\right]_{\mathbf{r}^{\prime}, \mathbf{r}} t_{\mathbf{r}^{\prime}}, \quad \mathbf{r} \in \Re_{\mathrm{tw}} .
\end{aligned}
$$

\subsection{Monodromy of the asymptotical operator}

Recall the notation from Section 2.3.1. Let us identify the space of linear operators $\operatorname{Hom}_{\mathbb{C}}\left(\mathbb{C}^{\mu}, H\right)$ with the space of square matrices of size $\mu$ by fixing the following bases $\left\{\phi_{\mathbf{r}}(\mathbf{x}):=\mathbf{x}^{\mathbf{r}}\right\}_{\mathbf{r} \in \mathfrak{R}} \subset H$ and $\left\{e_{i}\right\}_{1 \leq i \leq \mu} \subset \mathbb{C}^{\mu}$, i.e.,

$$
\mathbb{A}\left(e_{i}\right)=\sum_{\mathbf{r} \in \mathfrak{R}}[\mathbb{A}]_{\mathbf{r}, i} \phi_{\mathbf{r}}
$$

The asymptotical operator $\Psi_{\mathbf{s}} R_{\mathbf{s}} e^{U_{\mathbf{s}} / z}$ can be viewed as a matrix with entries formal asymptotical series. Let us fix a loop $\gamma$ in $\Sigma$. We would like to find 
out how the operator changes under the analytic continuation along $\gamma$. The answer can be stated in the following way. Let $M(\gamma, t) \in \operatorname{Hom}_{\mathbb{C}}(H, H)$ be the operator whose matrix is

$$
[M(\gamma, t)]_{\mathbf{r}^{\prime}, \mathbf{r}}=\frac{1}{c t_{\mathbf{m}}+d} \frac{\partial t_{\mathbf{r}^{\prime}}}{\partial \widetilde{t}_{\mathbf{r}}}-z c \delta_{\mathbf{m}, \mathbf{r}} \delta_{0, \mathbf{r}^{\prime}}
$$

where $c, d$, and $\widetilde{t}_{\mathbf{r}}$ are determined via $\gamma$ as it was stated in Corollary 3.2. Analytic continuation along $\gamma$ transforms the sequence of critical values $\left(u_{1}(\mathbf{s}), \ldots, u_{\mu}(\mathbf{s})\right)$ via some permutation $p$. Let us denote by $P(\gamma) \in$ $\operatorname{Hom}_{\mathbb{C}}\left(\mathbb{C}^{\mu}, \mathbb{C}^{\mu}\right)$ the linear operator whose matrix is given by

$$
[P(\gamma)]_{i, j}=\delta_{i, p(j)}
$$

Proposition 3.3. The analytic continuation along the loop $\gamma$ transforms the asymptotical operator $\Psi_{\mathrm{s}} R_{\mathrm{s}} e^{U_{\mathrm{s}} / z}$ into

$$
{ }^{\mathrm{T}} M(\gamma, t) \Psi_{\mathrm{s}} R_{\mathbf{s}} e^{U_{\mathrm{s}} / z} P(\gamma)
$$

where for a linear operator $\mathbb{A}: H \rightarrow H$ we denote by ${ }^{\mathrm{T}} \mathbb{A}$ the transpose of $\mathbb{A}$ with respect to the residue pairing $(\cdot, \cdot)$.

Proof. Let us denote by $I_{i}(\mathbf{s}, z)(1 \leq i \leq \mu)$ the stationary phase asymptotic of the oscillatory integral

$$
(-2 \pi z)^{-3 / 2} \int_{\mathcal{B}_{i}} e^{F(\mathbf{s}, \mathbf{x}) / z} d^{3} \mathbf{x} .
$$

By definition the asymptotical operator is defined by the following identity:

$$
\left(\phi_{\mathbf{r}}, \Psi_{\mathbf{s}} R_{\mathbf{s}} e^{U_{\mathbf{s}} / z} e_{i}\right)=z \frac{\partial}{\partial t_{\mathbf{r}}}\left(\frac{I_{i}(\mathbf{s}, z)}{\pi_{A}}\right), \quad \mathbf{r} \in \mathfrak{R}, \quad 1 \leq i \leq \mu .
$$

The analytic continuation along $\gamma$ transforms the above matrix into

$$
z \frac{\partial}{\partial \widetilde{t}_{\mathbf{r}}}\left(\frac{I_{p(i)}(\mathbf{s}, z)}{\pi_{A}\left(c t_{\mathbf{m}}+d\right)}\right)=\sum_{\mathbf{r}^{\prime} \in \mathfrak{R}} z \frac{\partial}{\partial t_{\mathbf{r}^{\prime}}}\left(\frac{I_{p(i)}(\mathbf{s}, z)}{\pi_{A}\left(c t_{\mathbf{m}}+d\right)}\right) \frac{\partial t_{\mathbf{r}}^{\prime}}{\partial \widetilde{t}_{\mathbf{r}}}
$$

Note that

$$
\sum_{\mathbf{r}^{\prime} \in \mathfrak{R}} z \frac{\partial}{\partial t_{\mathbf{r}^{\prime}}}\left(\frac{1}{c t_{\mathbf{m}}+d}\right) \frac{\partial t_{\mathbf{r}^{\prime}}}{\partial \widetilde{t}_{\mathbf{r}}}=z \frac{\partial t_{\mathbf{m}}}{\partial \widetilde{t}_{\mathbf{m}}}\left(\frac{-c}{\left(c t_{\mathbf{m}}+d\right)^{2}}\right) \delta_{\mathbf{m}, \mathbf{r}}=-z c \delta_{\mathbf{m}, \mathbf{r}}
$$


and

$$
\frac{I_{p(i)}(\mathbf{s}, z)}{\pi_{A}}=z \frac{\partial}{\partial t_{0}}\left(\frac{I_{p(i)}(\mathbf{s}, z)}{\pi_{A}}\right) .
$$

Hence the RHS of (25) becomes

$$
\sum_{\mathbf{r}^{\prime} \in \mathfrak{R}}\left(\frac{z}{c t_{\mathbf{m}}+d}\right) \frac{\partial t_{\mathbf{r}^{\prime}}}{\partial \widetilde{t}_{\mathbf{r}}} \frac{\partial}{\partial t_{r^{\prime}}}\left(\frac{I_{p(i)}(\mathbf{s}, z)}{\pi_{A}}\right)-\left(z c \delta_{\mathbf{m}, \mathbf{r}}\right) z \frac{\partial}{\partial t_{0}}\left(\frac{I_{p(i)}(\mathbf{s}, z)}{\pi_{A}}\right) .
$$

It remains only to check that the above expression coincides with

$$
\left(M(\gamma, t)\left(\phi_{\mathbf{r}}\right), \Psi_{\mathbf{s}} R_{\mathbf{s}} e^{U_{\mathbf{s}} / z} P e_{i}\right)=\sum_{\mathbf{r}^{\prime} \in \mathfrak{R}}[M(\gamma, t)]_{\mathbf{r}^{\prime}, \mathbf{r}} z \frac{\partial}{\partial t_{\mathbf{r}^{\prime}}}\left(\frac{I_{p(i)}(\mathbf{s}, z)}{\pi_{A}}\right) .
$$

Let us introduce the linear operators (cf. Corollary 3.1)

$$
J(\gamma, t): H \rightarrow H, \quad[J(\gamma, t)]_{\mathbf{r}^{\prime}, \mathbf{r}}=\frac{\partial t_{\mathbf{r}^{\prime}}}{\partial \widetilde{t}_{\mathbf{r}}}
$$

and

$$
X(\gamma, t): \mathcal{H} \rightarrow \mathcal{H}, \quad X(\gamma, t)=1-\left(\frac{c z}{c t_{\mathbf{m}}+d}\right) \phi_{\mathbf{m}} \bullet \mathbf{s}=0,
$$

where $\phi_{\mathbf{m}} \bullet_{\mathbf{s}=0}: H \rightarrow H$ is the operator of multiplication by $\phi_{\mathbf{m}}$ in the Jacobi algebra $H$. Note that $X(\gamma, t)$ is a symplectic transformation.

Proposition 3.4. The analytic continuation along the loop $\gamma$ transforms the total ancestor potential $\mathscr{A}_{\mathbf{s}}(\hbar ; \mathbf{q})$ into

$$
\left((X(\gamma, t))^{\wedge} \mathscr{A}_{\mathbf{s}}\right)\left(\left(c t_{\mathbf{m}}+d\right)^{2} \hbar ; J(\gamma, t) \mathbf{q}\right)
$$

where we first apply the operator $(X(\gamma, t))^{\wedge}$ and then we rescale $\hbar$ and $\mathbf{q}$.

Proof. We may assume that $P(\gamma, t)=1$ because $P$ is a permutation matrix, so its quantization $(P)^{\wedge}$ will leave the product of Kontsevich-Witten tau functions invariant. Put $M=M_{0}+z M_{1}$. Then we have

$$
\begin{aligned}
{ }^{\mathrm{T}} M \Psi_{\mathbf{s}} R_{\mathbf{s}} e^{U_{\mathbf{s}} / z} & =\widetilde{\Psi}_{\mathbf{s}} \widetilde{R}_{\mathbf{s}} e^{U_{\mathbf{s}} / z}, \quad \text { where } \widetilde{\Psi}_{\mathbf{s}}=M_{0}^{-1} \Psi_{\mathbf{s}} \\
\widetilde{R}_{\mathbf{s}} & =\Psi_{\mathbf{s}}^{-1} M_{0}{ }^{\mathrm{T}} M \Psi_{\mathbf{s}} R_{\mathbf{s}}
\end{aligned}
$$

The quantization is in general only a projective representation. However, the quantization of the operators $\Psi_{\mathrm{s}}^{-1} M_{0}^{\mathrm{T}} M \Psi_{\mathrm{s}}$ and $R_{\mathrm{s}}$ involves quantizing 
only $p^{2}$ and $p q$-terms. Since the cocycle (13) on such terms vanishes we get

$$
\left(\widetilde{R}_{\mathbf{s}}\right)^{\wedge}=\left(\Psi_{\mathbf{s}}^{-1} M_{0}{ }^{\mathrm{T}} M \Psi_{\mathbf{s}}\right)^{\wedge}\left(R_{\mathbf{s}}\right)^{\wedge}
$$

The operators $M_{0}$ and $\Psi_{\mathrm{s}}$ are independent of $z$ and their quantizations by definition are just changes of variables. Hence

$$
\left(\widetilde{\Psi}_{\mathbf{s}} \widetilde{R}_{\mathbf{s}}\right)^{\wedge}=\left(M_{0}^{-1}\right)^{\wedge}\left(M_{0}{ }^{\mathrm{T}} M\right)^{\wedge}\left(\Psi_{\mathrm{s}} R_{\mathrm{s}}\right)^{\wedge}
$$

By definition $\Delta_{i}^{-1}$ is $\left(\partial_{u_{i}}, \partial_{u_{i}}\right)$, which gains a factor of $\left(c t_{\mathbf{m}}+d\right)^{-2}$ under analytic continuation. The ancestor potential (17) is transformed into

$$
\left(M_{0}^{-1}\right)^{\wedge}\left(M_{0}{ }^{\mathrm{T}} M\right)^{\wedge}\left(\mathscr{A}_{\mathbf{s}}\left(\left(c t_{\mathbf{m}}+d\right)^{2} \hbar ;\left(c t_{\mathbf{m}}+d\right) \mathbf{q}\right)\right)
$$

Note that

$$
M_{0}^{-1}=\left(c t_{\mathbf{m}}+d\right) J(\gamma, t)^{-1}, \quad M_{0}\left({ }^{\mathrm{T}} M\right)=X(\gamma, t)
$$

It remains only to notice that the rescaling

$$
(\hbar, \mathbf{q}) \mapsto\left(\left(c t_{\mathbf{m}}+d\right)^{2} \hbar,\left(c t_{\mathbf{m}}+d\right) \mathbf{q}\right)
$$

commutes with the action of any quantized operator.

\subsection{Quasi-modularity}

It is known that the ancestor potential $\mathscr{A}_{\mathbf{s}}$ depends analytically on $\mathbf{s} \in D$, where $D \subset \mathcal{S}$ is any open domain in which the primitive form is singlevalued. In particular for each fixed $\sigma \in \Sigma$, we can take the limit

$$
\mathscr{A}_{\sigma}(\hbar ; \mathbf{q}):=\lim _{\substack{t_{\mathbf{r}} \rightarrow 0 ; \\ \mathbf{r} \in \mathfrak{R} \backslash\{\mathbf{m}\}}} \mathscr{A}_{\mathbf{S}}(\hbar ; \mathbf{q})
$$

For the proof of the above statement see [15], or more generally [14].

The marginal flat coordinate can be written as

$$
t_{\mathbf{m}}=\frac{a^{\prime} \tau+b^{\prime}}{c^{\prime} \tau+d^{\prime}}, \quad \tau \in \mathbb{H}, \quad\left[\begin{array}{ll}
a^{\prime} & b^{\prime} \\
c^{\prime} & d^{\prime}
\end{array}\right] \in \mathrm{SL}_{2}(\mathbb{C}) .
$$


We define

$$
\bar{t}_{\mathbf{m}}:=\frac{a^{\prime} \bar{\tau}+b^{\prime}}{c^{\prime} \bar{\tau}+d^{\prime}}
$$

where $\bar{\tau}$ is the standard conjugation in the upper half-plane $\mathbb{H}$. Since the analytic continuation transforms $\tau$ and $\bar{\tau}$ via the same fractional linear transformation, we get that the analytic transformation along $\gamma$ transforms $t_{\mathbf{m}}$ and $\bar{t}_{\mathbf{m}}$, respectively, into

$$
t_{\mathbf{m}} \mapsto \frac{a t_{\mathbf{m}}+b}{c t_{\mathbf{m}}+d} \quad \text { and } \quad \bar{t}_{\mathbf{m}} \mapsto \frac{a \bar{t}_{\mathbf{m}}+b}{c \bar{t}_{\mathbf{m}}+d} .
$$

A direct computation shows that

$$
-\frac{1}{t_{\mathbf{m}}-\bar{t}_{\mathbf{m}}} \mapsto-\frac{\left(c t_{\mathbf{m}}+d\right)^{2}}{t_{\mathbf{m}}-\bar{t}_{\mathbf{m}}}+c\left(c t_{\mathbf{m}}+d\right) .
$$

Following [15], we define anti-holomorphic completion of the ancestor potential

$$
\widetilde{\mathscr{A}_{\sigma}}(\hbar ; \mathbf{q})=(\widetilde{X}(\sigma, z))^{\wedge} \mathscr{A}_{\sigma}(\hbar ; \mathbf{q})
$$

where

$$
\widetilde{X}(\sigma, z)=1-\left(\frac{z}{t_{\mathbf{m}}-\bar{t}_{\mathbf{m}}}\right) \phi_{\mathbf{m}} \bullet_{\mathbf{s}=0} .
$$

Proposition 3.4 yields the following corollary (cf. [15]).

Corollary 3.5. The analytic continuation along $\gamma$ transforms the modified total ancestor potential as follows:

$$
\widetilde{\mathscr{A}_{\sigma}}(\hbar ; \mathbf{q}) \mapsto \widetilde{\mathscr{A}_{\sigma}}\left(\left(c t_{\mathbf{m}}+d\right)^{2} \hbar ; J(\gamma, \sigma) \mathbf{q}\right)
$$

Proof of Theorem 1.1. Assume that $\gamma \in \Gamma(W)$, then $\rho_{\neq 0}(\gamma)=J^{n}$ for some integer $n$, where $J$ is the classical monodromy operator. Since $J\left(\alpha_{\mathbf{r}}\right)=$ $e^{2 \pi \sqrt{-1} \operatorname{deg}\left(\mathbf{x}^{\mathbf{r}}\right)} \alpha_{\mathbf{r}}$, we get that the matrix of $J(\gamma, \sigma)$ is diagonal and we have

$$
[J(\gamma, \sigma)]_{\mathbf{r}, \mathbf{r}}=\left(c t_{\mathbf{m}}+d\right) e^{2 \pi \sqrt{-1} n \operatorname{deg}\left(\mathbf{x}^{\mathbf{r}}\right)}, \quad \mathbf{r} \in \mathfrak{R}_{\mathrm{tw}}
$$

and

$$
[J(\gamma, \sigma)]_{\mathbf{m}, \mathbf{m}}=\left(c t_{\mathbf{m}}+d\right)^{2}, \quad[J(\gamma, \sigma)]_{0,0}=1
$$


The total ancestor potential is invariant under the rescaling $q_{k}^{\mathbf{r}} \mapsto$ $e^{2 \pi \sqrt{-1} n \operatorname{deg}\left(\mathbf{x}^{\mathbf{r}}\right)} q_{k}^{\mathbf{r}}$, because it is quasi-homegeneous. Hence we may assume that $n=0$. The statement of the theorem follows from Corollary 3.5 by comparing the coefficients of the monomials in $\mathbf{q}$ and $\hbar$. Each coefficient $\widetilde{c}\left(t_{\mathbf{m}}\right)$ of the modified potential has the form

$$
\widetilde{c}\left(t_{\mathbf{m}}\right)=\sum_{i=0}^{\infty} \frac{c_{i}\left(t_{\mathbf{m}}\right)}{\left(t_{\mathbf{m}}-\bar{t}_{\mathbf{m}}\right)^{i}},
$$

where $c_{i}=0$ for $i \gg 0$. The analytical continuation of $\widetilde{c}\left(t_{\mathbf{m}}\right)$ is

$$
\widetilde{c}\left(\frac{a t_{\mathbf{m}}+b}{c t_{\mathbf{m}}+d}\right)=\left(c t_{\mathbf{m}}+d\right)^{w} \widetilde{c}\left(t_{\mathbf{m}}\right),
$$

where the identity follows from Corollary 3.5 and $w$ is a non-negative integer depending on the monomial (see [15] for more details).

\section{The modular groups $\Gamma(W)$}

The computation of $\Gamma(W)$ amounts to computing the monodromy group of several hypergeometric equations of the type

$$
x(1-x) y^{\prime \prime}(x)+(\gamma-(1+\alpha+\beta) x) y^{\prime}(x)-\alpha \beta y(x)=0,
$$

where $\alpha, \beta$, and $\gamma$ are positive rational numbers. Let us begin by briefly reviewing the main steps in the computation.

\subsection{Monodromy of the hypergeometric equations}

There are two cases which are used in our work.

4.1.1. The resonance case. We assume that $\gamma=\alpha+\beta=1-1 / \ell$, where $\ell$ is a positive integer. Near $x=0$ the hypergeometric equation (27) admits the following basis of solutions:

$$
\left\{\begin{array}{l}
F_{1}^{(0)}(x)=\frac{\Gamma(\alpha) \Gamma(\beta)}{\Gamma(\alpha+\beta)}{ }_{2} F_{1}(\alpha, \beta ; \gamma ; x) \\
F_{2}^{(0)}(x)=\frac{\Gamma(1-\alpha) \Gamma(1-\beta)}{\Gamma(2-\alpha-\beta)}{ }_{2} F_{1}(1-\alpha, 1-\beta ; 2-\gamma ; x) x^{1-\alpha-\beta} .
\end{array}\right.
$$


Near $x=1$ a basis of solutions is given by

$$
\left\{\begin{array}{l}
F_{1}^{(1)}(x)={ }_{2} F_{1}(\alpha, \beta ; 1 ; 1-x), \\
F_{2}^{(1)}(x)={ }_{2} F_{1}(\alpha, \beta ; 1 ; 1-x) \ln (1-x)+\sum_{n=1}^{\infty} b_{n}(1-x)^{n}
\end{array}\right.
$$

where

$$
\begin{aligned}
b_{n}= & \frac{(\alpha)_{n}(\beta)_{n}}{(n !)^{2}}\left(\frac{1}{\alpha}+\cdots+\frac{1}{\alpha+n-1}+\frac{1}{\beta}+\cdots+\frac{1}{\beta+n-1}\right. \\
& \left.-2\left(\frac{1}{1}+\cdots+\frac{1}{n}\right)\right) .
\end{aligned}
$$

Let us denote by $F^{(a)}(x)$ the column vector with entries $F_{1}^{(a)}(x)$ and $F_{2}^{(a)}(x)$ for $a=0,1$; then the local monodromy around $x=0$ acts as

$$
F^{(0)}(x) \mapsto M_{0}^{T} F^{(0)}(x) ; \quad M_{0}:=\left[\begin{array}{cc}
1 & 0 \\
0 & e^{2 \pi i(1-\alpha-\beta)}
\end{array}\right]
$$

It follows that the local monodromy around $x=1$ is given by

$$
F^{(1)}(x) \mapsto M_{1}^{T} F^{(1)}(x) ; \quad M_{1}:=\left[\begin{array}{cc}
1 & 2 \pi i \\
0 & 1
\end{array}\right]
$$

The series $F^{(0)}(x)$ and $F^{(1)}(x)$ are convergent in the region $\{x \in \mathbb{C}:|x|<$ $1,|1-x|<1\}$ and since they both provide a basis of solutions for the same differential equation, there is a constant matrix $C^{01}$, called connection matrix, s.t.,

$$
F^{(0)}(x)=C^{01} F^{(1)}(x)
$$

Let $\psi(z)=\Gamma^{\prime}(z) / \Gamma(z)$ be the digamma function. Put

$(30) \quad K_{1}=2 \psi(1)-\psi(\alpha)-\psi(\beta), \quad K_{2}=2 \psi(1)-\psi(1-\alpha)-\psi(1-\beta)$.

The key to the monodromy computation is the following lemma (see [1]). 
Lemma 4.1. The connection matrix is given by the following formula:

$$
C^{01}:=\left[\begin{array}{cc}
K_{1} & -1 \\
K_{2} & -1
\end{array}\right]
$$

provided the branch of $F^{(1)}(x)$ near $x=1$ is chosen appropriately.

Proof. The first row of $C^{01}$ directly follows from the formula 15.3.10 in [1]. The second row follows from the first row and the hypergeometric identity

$$
\begin{aligned}
& \frac{\Gamma(\alpha-\gamma+1) \Gamma(\beta-\gamma+1) \Gamma(\gamma-1)}{\Gamma(\alpha) \Gamma(\beta) \Gamma(1-\gamma)}{ }_{2} F_{1}(\alpha-\gamma+1, \beta-\gamma+1 ; 2-\gamma ; x) x^{1-\gamma} \\
& =-{ }_{2} F_{1}(\alpha, \beta ; \gamma ; x)+\frac{\Gamma(\alpha-\gamma+1) \Gamma(\beta-\gamma+1)}{\Gamma(1-\gamma) \Gamma(\alpha+\beta-\gamma+1)} \\
& \quad \times{ }_{2} F_{1}(\alpha, \beta ; \alpha+\beta-\gamma+1 ; 1-x)
\end{aligned}
$$

The local monodromies $M_{0}, M_{1}$, and the connection matrix $C^{01}$ completely determine the monodromy representation.

4.1.2. The non-resonance case. Now we assume that none of the exponents

$$
\lambda_{0}=1-\gamma, \quad \lambda_{1}=\gamma-\alpha-\beta, \quad \lambda_{\infty}=\beta-\alpha
$$

is an integer. Then we fix the following solutions. Near $x=0$ :

$$
\left\{\begin{array}{l}
F_{1}^{(0)}(x)={ }_{2} F_{1}(\alpha, \beta ; \gamma ; x) \\
F_{2}^{(0)}(x)={ }_{2} F_{1}(\alpha-\gamma+1, \beta-\gamma+1 ; 2-\gamma ; x) x^{1-\gamma}
\end{array}\right.
$$

Near $x=1$ :

$$
\left\{\begin{array}{l}
F_{1}^{(1)}(x)={ }_{2} F_{1}(\alpha, \beta ; \alpha+\beta-\gamma+1 ; 1-x) \\
F_{2}^{(1)}(x)={ }_{2} F_{1}(\gamma-\alpha, \gamma-\beta ; \gamma-\alpha-\beta+1 ; 1-x)(1-x)^{\gamma-\alpha-\beta}
\end{array}\right.
$$

Let us denote by $F^{(a)}(x)(a=0,1)$ the corresponding column vectors. Then the key fact is the following. Just like in the previous case, there is a common contractible domain where both $F^{(0)}(x)$ and $F^{(1)}(x)$ are convergent and hence one can define a connection matrix $D^{01}$, s.t., $F^{(0)}(x)=D^{01} F^{(1)}(x)$. 
Lemma 4.2. The connection matrices are given by the following formulas:

$$
D^{01}=\left[\begin{array}{ll}
\frac{\Gamma(\gamma) \Gamma(\gamma-\alpha-\beta)}{\Gamma(\gamma-\alpha) \Gamma(\gamma-\beta)} & \frac{\Gamma(\gamma) \Gamma(\alpha+\beta-\gamma)}{\Gamma(\alpha) \Gamma(\beta)} \\
\frac{\Gamma(2-\gamma) \Gamma(\gamma-\alpha-\beta)}{\Gamma(1-\alpha) \Gamma(1-\beta)} & \frac{\Gamma(2-\gamma) \Gamma(\alpha+\beta-\gamma)}{\Gamma(1+\alpha-\gamma) \Gamma(1+\beta-\gamma)}
\end{array}\right]
$$

\subsection{The group $\widetilde{\Gamma}(W)$}

Recall that $\widetilde{\Gamma}(W)=\operatorname{Im}\left(\rho_{0}\right)$, where

$$
\rho_{0}: \pi_{1}(\Sigma) \rightarrow \operatorname{GL}\left(\mathfrak{h}_{0}\right), \quad \mathfrak{h}_{0}=H_{2}\left(X_{0,1} ; \mathbb{C}\right)^{J} \cong H_{1}\left(E_{0} ; \mathbb{C}\right)
$$

is the $J$-invariant part of the monodromy representation. Given a basis $\{A, B\}$ of $\mathfrak{h}_{0}$, we can compute the monodromy action on that basis by computing the monodromy under analytic continuation of the corresponding period integrals $\pi_{A}(\sigma)$ and $\pi_{B}(\sigma)$ (see Section 2.2). Let $D=\sigma \partial_{\sigma}$, then both $\pi_{A}(\sigma)$ and $\pi_{B}(\sigma)$ satisfy a second-order differential equation

$$
D(D-1) \pi(\sigma)-C \sigma^{\ell}(D+\ell \alpha)(D+\ell \beta) \pi(\sigma)=0
$$

where $C$ is some constant, $\ell \in \mathbb{Z}, \alpha, \beta \in \mathbb{Q}$ and $\alpha+\beta=1-\frac{1}{\ell}$.

Using the substitution $x=C \sigma^{\ell}$, the differential equation (33) becomes the standard hypergeometric equation (27) (with $\gamma=\alpha+\beta$ ), so the monodromy can be computed as explained above. We fix a reference point on $\mathbb{C}-\{0,1, \infty\}$ near $x=1$ and a basis of solutions as (29). Denote by $M_{0}^{\mathrm{HG}}$ and $M_{1}^{\mathrm{HG}}$ the monodromy transformations of the column vector $F^{(1)}(x)$ corresponding to paths going around $x=0$ and $x=1$, i.e., $F^{(1)}(x) \mapsto M_{a}^{\mathrm{HG}} F^{(1)}$ $(x), a=0,1$. The superscript HG stands for hyper geometric reflecting the fact that $M_{i}^{\mathrm{HG}}$ are monodromy transformations of a hypergeometric equation. We may choose the paths, s.t.,

$$
M_{1}^{\mathrm{HG}}=M_{1}^{T} ; \quad M_{0}^{\mathrm{HG}}=\left(C^{01}\right)^{-1} M_{0}^{T} C^{01},
$$

where $M_{0}, M_{1}$ and $C^{01}$ are, respectively, the local monodromies near $x=$ 0,1 and the matrix giving the analytic continuation from $x=1$ to $x=0$ (see Lemma 4.1).

Our substitution $x=C \sigma^{\ell}$ is a covering $\Sigma-\{0\} \rightarrow \mathbb{C}-\{0,1, \infty\}$ of degree $\ell$. Let us denote by $M_{i}^{\mathrm{PF}}, 1 \leq i \leq \ell$, the monodromy transformations corresponding to loops going around $\sigma=p_{i}$, which are the singularities of 


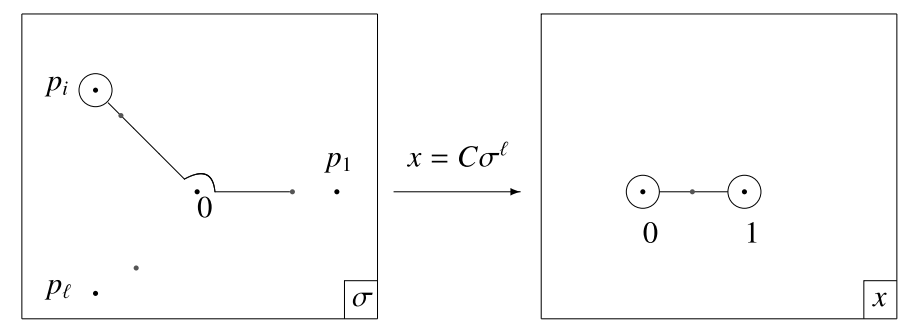

Figure 1: The path corresponding to $M_{i}^{\mathrm{PF}}$.

the differential equation (33),

$$
p_{i}=C^{-1 / \ell} \eta^{i}, \quad \eta=\exp (2 \pi \sqrt{-1} / \ell) .
$$

The superscript $P F$ stands for Picard-Fuchs reflecting the fact that $M_{i_{\sim}}^{\mathrm{PF}}$ are monodromies of a Picard-Fuchs equation. Since $\Sigma=\mathbb{C} \backslash\left\{p_{1}, \ldots, p_{\ell}\right\}, \widetilde{\Gamma}(W)$ is generated by $M_{i}^{\mathrm{PF}}, 1 \leq i \leq \ell$. Lifting the reference point and choosing the loops appropriately, we can arrange that

$$
M_{i}^{\mathrm{PF}}=\left(M_{0}^{\mathrm{HG}}\right)^{i-1} M_{1}^{\mathrm{HG}}\left(M_{0}^{\mathrm{HG}}\right)^{1-i}, \quad 1 \leq i \leq \ell .
$$

The path corresponding to $M_{i}^{\mathrm{PF}}$ is depicted in Figure 1: the red point in the $x$-plane is the reference point while the red points in the $\sigma$-plane are its lifts. Note that the portion of the path in the $\sigma$-plane between the two red points is the lift of the loop in the $x$-plane that goes $i-1$ times around $x=0$.

It remains only to explain how to find a basis of solutions $\left\{F_{1}^{\mathrm{GW}}, F_{2}^{\mathrm{GW}}\right\}$ that corresponds to periods of the elliptic curve, i.e.,

$$
F_{1}^{\mathrm{GW}}(\sigma)=\pi_{A}(\sigma), \quad F_{2}^{\mathrm{GW}}(\sigma)=\pi_{B}(\sigma),
$$

where $\{A, B\}$ is an integral basis of $H_{1}\left(E_{0} ; \mathbb{Z}\right)$, s.t. $A \circ B=1$.

Remark 4.3. The superscript $G W$ stands for Gromov-Witten and such a notation is motivated by the fact that the corresponding periods are known to be Gromov-Witten invariants (see [12, 15, 16] for more details).

The $j$-invariant of $E_{\sigma}$ has the form

$$
j(\sigma)=\frac{P(\sigma)}{\left(1-C \sigma^{\ell}\right)^{N}}, \quad P(\sigma) \in \mathbb{C}[\sigma],
$$


where the zeroes of the polynomial in the denominator are precisely the singular points in (35). Since we are interested in the matrices of the monodromy transformations, we have the freedom to rescale the above basis by any non-zero constant, so we may assume that

$$
\left[\begin{array}{l}
F_{1}^{\mathrm{GW}} \\
F_{2}^{\mathrm{GW}}
\end{array}\right]=K\left[\begin{array}{l}
F_{1}^{(1)} \\
F_{2}^{(1)}
\end{array}\right], \quad K=\left[\begin{array}{cc}
1 & 0 \\
-\frac{a}{b} & \frac{1}{b}
\end{array}\right],
$$

where $F_{1}^{(1)}, F_{2}^{(2)}$ are viewed as multi-valued functions of $\sigma$ via the substitution $x=C \sigma^{\ell}$, and $a$ and $b$ are some non-zero constants.

Lemma 4.4. The solutions $F_{i}^{\mathrm{GW}}, i=1,2$, correspond to periods of the elliptic curve (normalized as above) if and only if

$$
a=\frac{1}{N}\left(\ln P\left(p_{1}\right)+2 \pi \sqrt{-1} m\right), \quad b=2 \pi \sqrt{-1} / N
$$

where $p_{1}=C^{-1 / \ell}$ and $m$ is some integer.

Proof. Since $\tau:=F_{2}^{\mathrm{GW}} / F_{1}^{\mathrm{GW}}$ is the modulus of the elliptic curve, we must choose $a$ and $b$ in such a way that $j(\sigma)=1 / q+\cdots, q=e^{2 \pi i \tau}$. Inverting the relation (37) near $\sigma=p_{1}$, we find

$$
p_{1}-\sigma=\frac{e^{a}}{C \prod_{j=2}^{\ell}\left(p_{1}-p_{j}\right)} e^{b \tau}\left(1+O\left(e^{b \tau}\right)\right) .
$$

Then the lemma follows from

$$
j(\sigma)=\frac{P\left(p_{1}\right)}{e^{N(b \tau+a)}}+\cdots .
$$

Let us point out that the value of $a$ is fixed only up to $2 m \pi \sqrt{-1} / N$ for some $m \in \mathbb{Z}$. This corresponds to the fact that while there is a unique choice of an invariant cycle $A \in H_{1}\left(E_{\sigma} ; \mathbb{Z}\right)$ near $\sigma=p_{1}$, so that up to a constant $\pi_{A}(\sigma)$ agrees with $F_{1}^{\mathrm{GW}}$, for the second cycle $B \in H_{1}\left(E_{\sigma} ; \mathbb{Z}\right)$, we have the freedom to add any integer multiple of $A$. According to (34), (36) and (38) we have

Lemma 4.5. Let $F^{\mathrm{GW}}$ be the column vector with entries $F_{1}^{\mathrm{GW}}, F_{2}^{\mathrm{GW}}$. The monodromy transformation along the fixed loop going around $\sigma=p_{i}$ acts on 
$F^{\mathrm{GW}}$ by

$$
F^{\mathrm{GW}} \mapsto M_{i, 0}^{T} F^{\mathrm{GW}} ; \quad M_{i, 0}^{T}=K M_{i}^{\mathrm{PF}} K^{-1}
$$

As a consequnce, the group $\widetilde{\Gamma}(W)$ is isomorphic to the subgroup of $\mathrm{SL}_{2}(\mathbb{Z})$ generated by $M_{i, 0}, i=1, \ldots, \ell$.

Now we are in a position to prove Theorem 1.2. Some of the computations are quite cumbersome, so it is better to use some computer software, e.g., Mathematica or Maple.

\subsection{The Fermat $E_{6}^{(1,1)}$ case}

The polynomial in this case is

$$
f(\sigma, \mathbf{x})=x_{1}^{3}+x_{2}^{3}+x_{3}^{3}+\sigma x_{1} x_{2} x_{3}
$$

Its modular group is well known. For the sake of completeness, let us recall the computation. The $j$-invariant of this family is

$$
j(\sigma)=-\frac{\sigma^{3}\left(\sigma^{3}-216\right)^{3}}{\left(\sigma^{3}+27\right)^{3}} .
$$

The weights of Equation (33) are $\alpha=\beta=1 / 3, \gamma=2 / 3$. According to (39) we have

$$
\begin{aligned}
& M_{1,0}=\left[\begin{array}{ll}
1 & 3 \\
0 & 1
\end{array}\right], \quad M_{2,0}=\left[\begin{array}{cc}
1-3 m & 3 m^{2} \\
-3 & 1+3 m
\end{array}\right], \\
& M_{3,0}=\left[\begin{array}{cc}
4-3 m & 3(m-1)^{2} \\
-3 & 3 m-2
\end{array}\right]
\end{aligned}
$$

where the choice of $m$ depends on the coice of a symplectic basis $\{A, B\}$ in $H_{1}\left(E_{\sigma} ; \mathbb{Z}\right)$. The group generated by the matrices $M_{i, 0}$ is independent of the choice of $m$ and it coincides with $\Gamma(3)$, i.e., $\widetilde{\Gamma}(W)=\Gamma(3)$. For the period integrals $\Phi_{\mathbf{r}}(\sigma), \mathbf{r} \in \mathfrak{R}_{\mathrm{tw}}$, the monodromy acts on the corresponding flat sections by multiplication of $e^{2 \pi \sqrt{-1} \operatorname{deg} \phi_{\mathbf{r}}}$, which is just $J$ or $J^{2}$, with $J$ the classical monodromy operator. Thus $\Gamma(W)=\widetilde{\Gamma}(W)=\Gamma(3)$. 


\subsection{The Fermat $E_{7}^{(1,1)}$ case}

The polynomial in this case is

$$
f(\sigma, \mathbf{x})=x_{1}^{4}+x_{2}^{4}+x_{3}^{2}+\sigma x_{1}^{2} x_{2}^{2} .
$$

The $j$-invariant is

$$
j(\sigma)=16 \frac{\left(\sigma^{2}+12\right)^{3}}{\left(4-\sigma^{2}\right)^{2}} .
$$

Thus $C=1 / 4, \ell=2$ and $P(\sigma)=\left(\sigma^{2}+12\right)^{3}$.

4.4.1. Monodromy of the invariant part. The parameters of Equation (33) are $\alpha=\beta=1 / 4, \gamma=1 / 2$. We consider two singular points $p_{1}=2$ and $p_{2}=-2$. According to (39), the monodromy group is generated by the following two matrices:

$$
M_{1,0}=\left[\begin{array}{ll}
1 & 2 \\
0 & 1
\end{array}\right], \quad M_{2,0}=\left[\begin{array}{cc}
1-2 m & 2 m^{2} \\
-2 & 1+2 m
\end{array}\right]
$$

where the choice of $m$ depends on the coice of a symplectic basis $\{A, B\}$ in $H_{1}\left(E_{\sigma} ; \mathbb{Z}\right)$. We choose the basis to be such that $m=0$; then the monodromy group $\widetilde{\Gamma}(W)$ is the group of all matrices

$$
g=\left[\begin{array}{ll}
a & b \\
c & d
\end{array}\right] \in \mathrm{SL}_{2}(\mathbb{Z}), \quad a \equiv d \equiv 1(\bmod 4), \quad b \equiv c \equiv 0(\bmod 2) .
$$

This is an index 2 subgroup of $\Gamma(2)$. Note that in this case the matrix presentation of $\widetilde{\Gamma}(W)$ depends on the parity of $m$. Namely if $m$ is even; then we get the matrix group from above. If $m$ is odd then we obtain again an index 2 subgroup of $\Gamma(2)$ obtained from the above one by conjugation with the matrix $\left[\begin{array}{ll}1 & 1 \\ 0 & 1\end{array}\right]$.

4.4.2. Monodromy of the twisted sector. We fix the following basis of the twisted sectors in the Jacobian algebra $\mathscr{Q}_{W}$ :

$\phi_{\mathbf{r}}(\mathbf{x})=x_{1}^{r_{1}} x_{2}^{r_{2}} x_{3}^{r_{3}}, \quad \mathbf{r}=\{(100),(010),(200),(110),(020),(210),(120)\}$. 
According to Milanov and Shen [16], the corresponding period integrals $\Phi_{\mathbf{r}}(\sigma)$ satisfy

$$
\left(4-\sigma^{2}\right) \partial_{\sigma} \Phi_{\mathbf{r}}(\sigma)=2 \operatorname{deg}\left(\phi_{\mathbf{r}}\right) \sigma \Phi_{\mathbf{r}}(\sigma), \quad \mathbf{r} \neq(200),(020),
$$

and

$$
\left\{\begin{array}{l}
\left(4-\sigma^{2}\right) \partial_{\sigma} \Phi_{200}(\sigma)=\frac{\sigma}{2} \Phi_{200}(\sigma)-\Phi_{020}(\sigma) \\
\left(4-\sigma^{2}\right) \partial_{\sigma} \Phi_{020}(\sigma)=-\Phi_{200}(\sigma)+\frac{\sigma}{2} \Phi_{020}(\sigma)
\end{array}\right.
$$

The monodromy of Equations (40) around $\sigma=p_{i}$ is straightforward to compute:

$$
\Phi_{\mathbf{r}} \mapsto e^{2 \pi \sqrt{-1} \operatorname{deg}\left(\phi_{\mathbf{r}}\right)} \Phi_{\mathbf{r}}, \quad i=1,2 .
$$

For system (41), we can first obtain a second-order differential equation for $\Phi_{200}$, which after the substitution $x=\sigma^{2} / 4$ becomes the hypergeometric equation with weights $\alpha=3 / 4, \beta=1 / 4, \gamma=1 / 2$. It follows that the system can be solved as follows:

$$
\left[\begin{array}{l}
\Phi_{200} \\
\Phi_{020}
\end{array}\right]=\left[\begin{array}{cc}
F_{1}^{(1)} & F_{2}^{(1)} \\
L F_{1}^{(1)} & L F_{2}^{(1)}
\end{array}\right]\left[\begin{array}{c}
A_{200} \\
A_{020}
\end{array}\right]
$$

where $A_{200}$ and $A_{020}$ are flat sections of the vanishing cohomology bundle and

$$
L=-\left(4-\sigma^{2}\right) \partial_{\sigma}+\sigma / 2
$$

Let us denote by $M_{i, 1 / 4}$ the $2 \times 2$ matrix defined by the analytic continuation along a simple loop $\gamma_{i}$ around the point $\sigma=p_{i}$ of the following vector-valued function:

$$
F^{(1)} \mapsto M_{i, 1 / 4}^{T} F^{(1)}, \quad F^{(1)}=\left(F_{1}^{(1)}, F_{2}^{(1)}\right)^{T},
$$

where the reason to use the transposition operation ${ }^{T}$ will become clear shortly. Since the LHS of (42) consists of holomorphic sections of the 
vanishing cohomology bundle, it must be invariant under analytic continuation along any loop in $\Sigma$. It follows that the monodromy of the flat sections is

$$
\left[\begin{array}{c}
A_{200} \\
A_{020}
\end{array}\right] \mapsto\left(M_{i, 1 / 4}\right)^{-1}\left[\begin{array}{l}
A_{200} \\
A_{020}
\end{array}\right]
$$

Note that in the basis $\left\{\alpha_{200}, \alpha_{020}\right\}$ dual to $\left\{A_{200}, A_{020}\right\}$ the matrix of the monodromy transformation $\rho\left(\gamma_{i}\right)$ is precisely $M_{i, 1 / 4}$. On the other hand, arguing as in Section 4.2, we get

$$
M_{i, 1 / 4}=\left(\left(M_{0}^{\mathrm{HG}}\right)^{i-1} M_{1}^{\mathrm{HG}}\left(M_{0}^{\mathrm{HG}}\right)^{(1-i)}\right)^{T}, \quad i=1,2
$$

where $M_{0}^{\mathrm{HG}}$ and $M_{1}^{\mathrm{HG}}$ are the monodromies of the corresponding hypergeometric equation around $x=0$ and $x=1$, respectively. Let us denote by

$$
D_{0}=\left[\begin{array}{cc}
1 & 0 \\
0 & e^{2 \pi \sqrt{-1}(1-\gamma)}
\end{array}\right], \quad D_{1}=\left[\begin{array}{cc}
1 & 0 \\
0 & e^{2 \pi \sqrt{-1}(\gamma-\alpha-\beta)}
\end{array}\right]
$$

the local monodromies of the hypergeometric equation; then (see Lemma 4.2)

$$
M_{1}^{\mathrm{HG}}=D_{1}^{T} ; \quad M_{0}^{\mathrm{HG}}=\left(C^{01}\right)^{-1} D_{0}^{T} C^{01} .
$$

A straightforward computation yields

$$
M_{1,1 / 4}=\left[\begin{array}{cc}
1 & 0 \\
0 & -1
\end{array}\right], \quad M_{2,1 / 4}=\left[\begin{array}{cc}
-1 & 0 \\
0 & 1
\end{array}\right] \text {. }
$$

The group generated by these two matrices is $\mathbb{Z} / 2 \times \mathbb{Z} / 2$. Let us point out that this agrees with the so-called Schwarz list (see [13]), since the exponents of the hypergeometric equation are

$$
\lambda_{0}=1-\gamma=\frac{1}{2}, \quad \lambda_{1}=\gamma-\alpha-\beta=-\frac{1}{2}, \quad \lambda_{\infty}=\beta-\alpha=-\frac{1}{2} .
$$

Lemma 4.6. The kernel Ker $\left(\rho_{0}\right)=0$ and the modular group $\Gamma(W)=\Gamma(4)$.

Proof. Let $\widetilde{\Sigma} \rightarrow \Sigma$ be the universal cover of $\Sigma$ and let $\tau: \widetilde{\Sigma} \rightarrow \mathbb{H}$ be the map given by the quotient of two periods: $\tau=\pi_{B} / \pi_{A}$. Using the Picard-Fuchs 
equation we find that

$$
d \tau=\frac{\operatorname{Wr}\left(\pi_{A}, \pi_{B}\right)}{\pi_{A}^{2}} d \sigma \neq 0
$$

By the implicit function theorem $\tau$ is a local homeomorphism. Using the $j$ invariant we see that the map $\tau$ is finite and surjective. Finite maps between analytic varieties are proper. It follows that $\tau$ is a covering. Finally, since both $\widetilde{\Sigma}$ and $\mathbb{H}$ are simply connected, $\tau$ must be an isomorphism.

The monodromy group $\widetilde{\Gamma}(W)$ induces an action on $\mathbb{H}$. If we assume that $\operatorname{Ker}\left(\rho_{0}\right)$ is non-trivial; then we can find two different points on $\widetilde{\Sigma}$ such that their $\tau$-images coincide - contradiction. In particular, the condition (2) is satisfied and the map (3) is well defined. Moreover, using our computation of the monodromy of the twisted periods, we get that $\operatorname{Im}\left(\rho_{W}\right) \cong \mathbb{Z} / 2 \times \mathbb{Z} / 2$, i.e., we have a surjective group homomorphism

$$
\rho_{W}: \widetilde{\Gamma}(W) \longrightarrow \mathbb{Z} / 2 \times \mathbb{Z} / 2
$$

which implies that the index of $\operatorname{Ker} \rho_{W}$ in $\widetilde{\Gamma}(W)$ is 4 . On the other hand, it is easy to see that $\Gamma(4)$ can be generated by the following (see [18]) 6 elements:

$$
\left[\begin{array}{cc}
1 & -4 \\
0 & 1
\end{array}\right], \quad\left[\begin{array}{cc}
-3 & -4 \\
4 & 5
\end{array}\right],\left[\begin{array}{ll}
1 & 0 \\
4 & 1
\end{array}\right], \quad\left[\begin{array}{cc}
9 & -4 \\
16 & -7
\end{array}\right], \quad\left[\begin{array}{cc}
5 & -4 \\
4 & -3
\end{array}\right],\left[\begin{array}{cc}
9 & -16 \\
4 & -7
\end{array}\right],
$$

We leave it to the reader to check that each of these matrices is in the kernel of $\rho_{W}$. For example,

$$
\left[\begin{array}{cc}
-3 & -4 \\
4 & 5
\end{array}\right]=\left(\left(M_{2,0}\right)^{-1} M_{1,0}\right)^{2}
$$

Under $\rho_{W}$ the matrix $M_{i, 0}$ is mapped to $M_{i, 1 / 4}$. Using the explicit formulas for $M_{i, 1 / 4}$ we get that the above matrix is in the kernel of $\rho_{W}$. The remaining five matrices are treated in a similar fashion. Hence we have

$$
\Gamma(4) \subseteq \operatorname{Ker} \rho_{W} \subset \widetilde{\Gamma}(W) \subset \Gamma(2) \subset \mathrm{SL}_{2}(\mathbb{Z})
$$

Since the index of $\Gamma(2)$ in $\mathrm{SL}_{2}(\mathbb{Z})$ is 6 (see [17]), the index of $\operatorname{Ker}\left(\rho_{W}\right)$ in $\mathrm{SL}_{2}(\mathbb{Z})$ must be $4 \times 2 \times 6=48$. However, the index of $\Gamma(4)$ in $\mathrm{SL}_{2}(\mathbb{Z})$ is also 48. The Lemma follows. 


\subsection{The Fermat $E_{8}^{(1,1)}$ case}

The polynomial in this case is

$$
f(\sigma, \mathbf{x})=x_{1}^{6}+x_{2}^{3}+x_{3}^{2}+\sigma x_{1}^{4} x_{2} .
$$

The $j$-invariant is

$$
j(\sigma)=1728 \frac{4 \sigma^{3}}{4 \sigma^{3}+27} .
$$

Thus $C=-\frac{4}{27}, \ell=3$ and $P(\sigma)=256 \sigma^{3}$.

4.5.1. Monodromy of the invariant part. The parameters of the hypergeometric equation (27) are $\alpha=\frac{1}{12}, \beta=\frac{7}{12}, \gamma=\frac{2}{3}$.

The singular points are

$$
p_{i}=-3 \cdot 4^{-1 / 3} e^{2 \pi \sqrt{-1}(i-1) / 3}, \quad 1 \leq i \leq 3
$$

According to (39), the corresponding monodromy transformations are

$$
M_{1,0}=\left[\begin{array}{ll}
1 & 1 \\
0 & 1
\end{array}\right], \quad M_{2,0}=\left[\begin{array}{cc}
-m & (1+m)^{2} \\
-1 & 2+m
\end{array}\right], \quad M_{3,0}=\left[\begin{array}{cc}
1-m & m^{2} \\
-1 & 1+m
\end{array}\right],
$$

where the integer $m$ depends on the choice of a symplectic basis in $H_{1}\left(E_{\sigma_{0}}\right.$; $\mathbb{Z})$. We choose a basis such that $m=0$ and simply denote the matrices $M_{i, 0}$ by $M_{i}$. The above matrices generate the entire modular group, because it is well known that the matrices in $\mathrm{SL}_{2}(\mathbb{Z})$

$$
S:=\left[\begin{array}{ll}
1 & 1 \\
0 & 1
\end{array}\right]=M_{1}, \quad T:=\left[\begin{array}{cc}
0 & 1 \\
-1 & 0
\end{array}\right]=M_{3} M_{1} M_{3}=M_{1} M_{3} M_{1}
$$

are generators of $\mathrm{SL}_{2}(\mathbb{Z})$. It is known that $\mathrm{SL}_{2}(\mathbb{Z})$ has a presentation in terms of the free group on two generators $a$ and $b$ satisfying the relations

$$
a b a=b a b, \quad(a b a)^{4}=1 .
$$

It follows that $\operatorname{Ker}\left(\rho_{0}\right)$ is the normal subgroup of the free group on three generators $M_{1}, M_{2}$ and $M_{3}$ generated by the relations

$$
M_{1} M_{3} M_{1}=M_{3} M_{1} M_{3}, \quad\left(M_{1} M_{3} M_{1}\right)^{4}=1, \quad M_{1} M_{3}=M_{2} M_{1} .
$$


4.5.2. Monodromy of the twisted sector. We fix a basis of monomials $\phi_{\mathbf{r}}(\mathbf{x})=x_{1}^{r_{1}} x_{2}^{r_{2}} x_{3}^{r_{3}}$ in the twisted sector of $H$, where $\mathbf{r}=\left(r_{1}, r_{2}, r_{3}\right)$ is given by

$\mathbf{r}=\{(1,0,0),(2,0,0),(0,1,0),(1,1,0),(3,0,0),(2,1,0),(4,0,0),(5,0,0)\}$.

The Picard-Fuchs equations for $\Phi_{\mathbf{r}}(\sigma), \mathbf{r}=(1,0,0),(5,0,0)$ have order 1

$$
\left(27+4 \sigma^{3}\right) \partial_{\sigma} \Phi_{\mathbf{r}}=12 \sigma^{2} \operatorname{deg}\left(\phi_{\mathbf{r}}\right) \Phi_{\mathbf{r}}
$$

The monodromy of these equations is straightforward to compute:

$$
\Phi_{\mathbf{r}} \mapsto e^{2 \pi \sqrt{-1} \operatorname{deg}\left(\phi_{\mathbf{r}}\right)} \Phi_{\mathbf{r}}
$$

The remaining periods satisfy three systems of differential equations. Let us describe them and their monodromies. In all three cases, we use the substitution $x=-4 \sigma^{3} / 27$ to reduce the system to a hypergeometric equation and then the monodromy is computed in the same way as before. We recall from [16] that the first system is

$$
\left\{\begin{aligned}
\left(27+4 \sigma^{3}\right) \partial_{\sigma} \Phi_{k+2,0,0} & =-(k+3) \sigma^{2} \Phi_{k+2,0,0}-\frac{9(k+1)}{2} \Phi_{k, 1,0} \\
\left(27+4 \sigma^{3}\right) \partial_{\sigma} \Phi_{k, 1,0} & =\frac{3(k+3) \sigma}{2} \Phi_{k+2,0,0}-(k+1) \sigma^{2} \Phi_{k, 1,0}
\end{aligned}\right.
$$

The solutions to the system have the following form:

$$
\left[\begin{array}{c}
\Phi_{k+2,0,0} \\
\Phi_{k, 1,0}
\end{array}\right]=\left[\begin{array}{cc}
F_{1}^{(1)} & F_{2}^{(1)} \\
L_{k} F_{1}^{(1)} & L_{k} F_{2}^{(1)}
\end{array}\right]\left[\begin{array}{c}
A_{k+2,0,0} \\
A_{k, 1,0}
\end{array}\right]
$$

where $A_{k+2,0,0}$ and $A_{k, 1,0}$ are flat sections of the vanishing cohomology bundle and $L_{k}$ is a first-order differential operator,

$$
L_{k}:=\frac{2}{3(k+3) \sigma}\left(\left(27+4 \sigma^{4}\right) \partial_{\sigma} \Phi_{k, 1,0}+(k+1) \sigma^{2} \Phi_{k, 1,0}\right), \quad k=0,1,2
$$

The period $\Phi_{k+2,0,0}$ satisfies the hypergeometric equation with weights

$$
\begin{aligned}
\left(\alpha_{k}, \beta_{k}, \gamma_{k}\right) & =(1 / 4,3 / 4,2 / 3), \quad(1 / 3,5 / 6,2 / 3), \quad(5 / 12,11 / 12,2 / 3), \\
k & =0,1,2 .
\end{aligned}
$$

It is convenient to decompose $\mathfrak{h}_{\neq 0}$ into eigenspaces $\mathfrak{h}_{d}$ of the classical monodromy $J$, where the eigenvalue corresponding to $\mathfrak{h}_{d}$ is $e^{2 \pi \sqrt{-1} d}$. The 
monodromy representation $\rho_{\neq 0}$ splits accordingly into a direct sum of representations $\rho_{d}$ and we denote by $M_{i, d}=\left[\rho_{d}\left(\gamma_{i}\right)\right](i=1,2,3)$ the matrices of the monodromy representation in an appropriately chosen basis of $\mathfrak{h}_{d}$. The same argument as in the $E_{7}^{(1,1)}$-case gives that

$$
\begin{aligned}
& M_{1,1 / 3}=\left[\begin{array}{cc}
1 & 0 \\
0 & e^{-2 \pi \sqrt{-1} / 3}
\end{array}\right], \quad M_{2,1 / 3}=\left[\begin{array}{cc}
-\frac{i}{\sqrt{3}} & -\frac{i}{2 \sqrt{3}} \\
-2+\frac{2 i}{\sqrt{3}} & \frac{1}{2}-\frac{i}{2 \sqrt{3}}
\end{array}\right], \\
& M_{3,1 / 3}=\left[\begin{array}{cc}
-\frac{i}{\sqrt{3}} & -\frac{1}{4}+\frac{i}{4 \sqrt{3}} \\
-\frac{4 i}{\sqrt{3}} & \frac{1}{2}-\frac{i}{2 \sqrt{3}}
\end{array}\right] .
\end{aligned}
$$

Using computer software one can check that the group generated by these matrices is finite of order 24. Alternatively, since the exponents of the hypergeometric equation are

$$
\lambda_{0}=1-\gamma=\frac{1}{3}, \quad \lambda_{1}=\gamma-\alpha-\beta=\frac{1}{3}, \quad \lambda_{\infty}=\beta-\alpha=\frac{1}{2},
$$

we find that the hypergeometric equation is in the Schwarz list and that its monodromy group is known to be isomorphic to $A_{4} \times \mathbb{Z} / 2$. It has order 24 .

From the system for $\left(\Phi_{300}, \Phi_{110}\right)$ we get

$$
\begin{aligned}
M_{1,1 / 2}= & {\left[\begin{array}{cc}
1 & 0 \\
0 & e^{-2 \pi \sqrt{-1} / 2}
\end{array}\right], \quad M_{2,1 / 2}=\left[\begin{array}{cc}
-\frac{1}{2} & -\frac{i}{2 \sqrt{3}} \\
\frac{3 \sqrt{3} i}{2} & \frac{1}{2}
\end{array}\right], } \\
M_{3,1 / 2}= & {\left[\begin{array}{cc}
-\frac{1}{2} & \frac{i}{2 \sqrt{3}} \\
-\frac{3 \sqrt{3} i}{2} & \frac{1}{2}
\end{array}\right] . }
\end{aligned}
$$

It is easy to check that these matrices generate a group with six elements. Again, we can obtain this from the general theory, since the exponents are

$$
\lambda_{0}=1-\gamma=\frac{1}{3}, \quad \lambda_{1}=\gamma-\alpha-\beta=-\frac{1}{2}, \quad \lambda_{\infty}=\beta-\alpha=\frac{1}{2}
$$

and the hypergeometric equation is again in the Schwarz list. The monodromy group is known to be the dihedral group $D_{6}$. 
Finally, from the system for $\left(\Phi_{400}, \Phi_{210}\right)$ we get

$$
\begin{aligned}
& M_{1,2 / 3}=\left[\begin{array}{cc}
1 & 0 \\
0 & e^{-4 \pi \sqrt{-1} / 3}
\end{array}\right], \quad M_{2,2 / 3}=\left[\begin{array}{cc}
\frac{i}{\sqrt{3}} & -\frac{\sqrt{3} i}{8} \\
\frac{8}{3}+\frac{8 \sqrt{3} i}{9} & \frac{1}{2}+\frac{i}{2 \sqrt{3}}
\end{array}\right], \\
& M_{3,2 / 3}=\left[\begin{array}{cc}
\frac{i}{\sqrt{3}} & \frac{3}{16}+\frac{\sqrt{3} i}{16} \\
-\frac{16 i}{3 \sqrt{3}} & \frac{1}{2}+\frac{\sqrt{3} i}{6}
\end{array}\right] .
\end{aligned}
$$

Again the matrices generate a group of order 24, which can also be proved by the general theory, since the exponents are

$$
\lambda_{0}=1-\gamma=\frac{1}{3}, \quad \lambda_{1}=\gamma-\alpha-\beta=-\frac{2}{3}, \quad \lambda_{\infty}=\beta-\alpha=\frac{1}{2} .
$$

Again, the monodromy group is isomorphic to $A_{4} \times \mathbb{Z} / 2$. One can check that (45)

$$
M_{1,2 / 3}^{-1}=S M_{1,1 / 3} S^{-1}, \quad M_{2,2 / 3}^{-1}=S M_{3,1 / 3} S^{-1}, \quad M_{3,2 / 3}^{-1}=S M_{2,1 / 3} S^{-1},
$$

where $S$ is the diagonal matrix with diagonal entries $-4 / 3$ and 1 .

Lemma 4.7. The condition (2) is satisfied and $\Gamma(W)=\Gamma(6)$.

Proof. For the first part of the Lemma, it is enough to check that the matrices $M_{i, d}, 1 \leq i \leq 3, d=1 / 3,1 / 2$ satisfy the relations (43). Note that due to (45) $M_{i, 2 / 3}$ also satisfy the relations. This is a straightforward computation, which we omit.

The homomorphism $\rho_{W}$ decomposes naturally into $\left(\rho_{W, 1 / 3}, \rho_{W, 1 / 2}\right.$, $\left.\rho_{W, 2 / 3}\right)$, where

$$
\rho_{W, d}: \widetilde{\Gamma}(W) \rightarrow \operatorname{GL}\left(\mathfrak{h}_{d}\right) / \mu_{6}, \quad d=1 / 3,1 / 2,2 / 3
$$

We claim that $\operatorname{Ker}\left(\rho_{W, 1 / 3}\right)=\operatorname{Ker}\left(\rho_{W, 2 / 3}\right)=\Gamma(3)$. It is easy to find that $\Gamma(3)$ is generated by the following matrices:

$$
\left[\begin{array}{ll}
1 & 3 \\
0 & 1
\end{array}\right], \quad\left[\begin{array}{cc}
1 & 0 \\
-3 & 1
\end{array}\right], \quad\left[\begin{array}{ll}
-2 & 3 \\
-3 & 4
\end{array}\right] .
$$


We leave it to the reader to check that they belong to the kernel of $\rho_{W, d}$ for $d=1 / 3$ and $d=2 / 3$. Our claim follows, because the index of $\Gamma(3)$ in $\mathrm{SL}_{2}(\mathbb{Z})$ is 24 (see [17]). Similarly one can verify that $\operatorname{Ker}\left(\rho_{W, 1 / 2}\right)=\Gamma(2)$, so we get

$$
\begin{aligned}
\Gamma(W) & =\operatorname{Ker}\left(\rho_{W}\right)=\operatorname{Ker}\left(\rho_{W, 1 / 3}\right) \cap \operatorname{Ker}\left(\rho_{W, 1 / 2}\right) \cap \operatorname{Ker}\left(\rho_{W, 2 / 3}\right) \\
& =\Gamma(3) \cap \Gamma(2)=\Gamma(6) .
\end{aligned}
$$

\section{Acknowledgments}

We thank Yongbin Ruan for his insight and support for this project. Both authors thank for many stimulating conversations. The first author benefited from conversations with Satoshi Kondo and Charles Siegel. We thank Arthur Greenspoon and Noriko Yui for editorial assistance. The work of both authors is supported by Grant-In-Aid and by the World Premier International Research Center Initiative (WPI Initiative), MEXT, Japan.

\section{References}

[1] M. Abramowitz and I.A. Stegun, Handbook of mathematical functions, U.S. Government Prinitng Office, Washington, DC, 1972.

[2] V. Arnold, S. Gusein-Zade and A. Varchenko, Singularities of differentiable maps. II Monodromy and Asymptotics of Integrals, Birkhäuser Boston, Boston, MA 1988. viii+492 pp.

[3] W. Chen and Y. Ruan, Orbifold Gromov-Witten theory, Orbifolds in mathematics and physics, Contemp. Math., 310, Amer. Math. Soc., Providence, RI, 2002, 25-85.

[4] A. Chiodo and Y. Ruan, Landau-Ginzburg/Calabi-Yau correspondence for quintic three-folds via symplectic transformations, Invent. Math. 182(1) (2010), 117-165.

[5] A. Chiodo and Y. Ruan, A global mirror symmetry framework for the Landau-Ginzburg/Calabi-Yau correspondence, Ann. Inst. Fourier (Grenoble) 61(7) (2011), 2803-2864.

[6] B. Dubrovin, Geometry of 2d Topological Field Theories, Integrable Systems and Quantum Groups. Lecture Notes in Maths., 1620: Springer, Berlin, 1996, 120-348.

[7] H. Fan, T.J. Jarvis and Y. Ruan, The Witten equation, mirror symmetry and quantum singularity theory, Ann. Math. 178(1) (2013), 1-106. 
[8] H. Fan, T.J. Jarvis and Y. Ruan, The Witten equation and its virtual fundamental cycle, Book in preparation, Preprint 2007, arXiv:0712.4025v3.

[9] A.B. Givental, Semisimple Frobenius structures at higher genus, Int. Math. Res. Notices 23 (2001), 1265-1286.

[10] A.B. Givental, Gromov-Witten invariants and quantization of quadratic Hamiltonians, Dedicated to the memory of I.G. Petrovskii on the occasion of his 100th anniversary, Mosc. Math. J. 1(4) (2001), 551-568, 645 .

[11] C. Hertling, Frobenius manifolds and moduli spaces for singularities, Cambridge Tracts in Mathematics, 151, Cambridge University Press, Cambridge, 2002, $\mathrm{x}+270$ pp.

[12] M. Krawitz and Y. Shen, Landau-Ginzburg/Calabi-Yau correspondence of all genera for elliptic orbifold $\mathbb{P}^{1}, 2011$, arXiv:1106.6270.

[13] M. Matsuda, Lectures on algebraic solutions of hypergeometric differential equations, Lect. Math. 15, Tokyo, 1985.

[14] T. Milanov, Analyticity of the total ancestor potential in singularity theory, Adv. Math., 255 (2014), 217-241.

[15] T. Milanov and Y. Ruan, Gromov-Witten theory of elliptic orbifold $\mathbb{P}^{1}$ and quasi-modular forms, preprint arXiv:1106.2321v1.

[16] T. Milanov and Y. Shen, Global mirror symmetry for invertible simple elliptic singularities, 2012, preprint arXiv:1210.6862.

[17] T. Miyake, Modular forms, Springer-Verlag, Berlin, 1989. x+335 pp.

[18] D. Mumford, Tata lectures on Theta, Birkhüser Basel, 1990. $\mathrm{XVI}+236 \mathrm{p}$.

[19] Y. Ruan, The Witten equation and the geometry of the LandauGinzburg model, String-Math 2011, 209-240, Proc. Symp. Pure Math., 85, Amer. Math. Soc., Providence, RI, 2012.

[20] K. Saito, On Periods of Primitive Integrals, I, preprint RIMS, 1982.

[21] K. Saito, Einfach-elliptische Singularitäten. Invent. Math. 23 (1974), 289-325.

[22] K. Saito, Extended affine root systems I. Coxeter transformations. Publ. RIMS, Kyoto Univ. 21 (1985), 75-179. 
[23] K. Saito and A. Takahashi, From primitive forms to Frobenius manifolds, Proc. Sympos. Pure Math. 78 (2008), 31-48.

[24] M. Saito, On the structure of Brieskorn lattice, Ann. Inst. Fourier 39 (1989), 27-72.

Kavli Institute for the Physics And Mathematics of the Universe (WPI)

Todai Institutes for Advanced Study, The University of Tokyo

Kashiwa, Chiba 277-8583

JAPAN

E-mail address: todor.milanov@ipmu.jp

E-mail address: yefeng.shen@ipmu.jp

RECEIVED JANUARY 9, 2014 